\title{
Asymmetric Total Synthesis of Dibenzocyclooctadiene Lignan Natural Products
}

\author{
Robert S. Coleman*, Srinivas Reddy Gurrala, Soumya Mitra, and Amresh Raao
}

Department of Chemistry, The Ohio State University, 100 West $18^{\text {th }}$ Avenue, Columbus, Ohio 43210

\section{Supporting Information}

\section{List of Contents:}

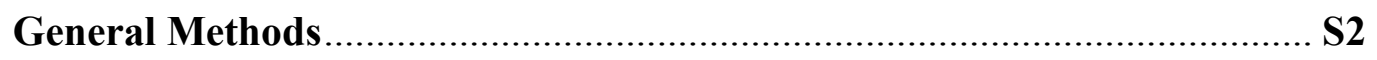

Experimental Procedures and Spectral Data ..................................S2-S18

X-ray Crystal Structures of 44, 47, and 51 .......................................... S19

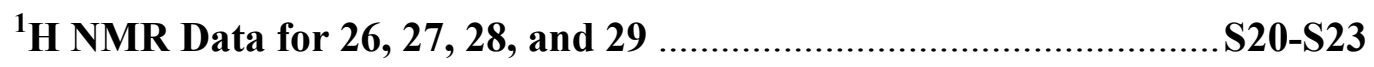

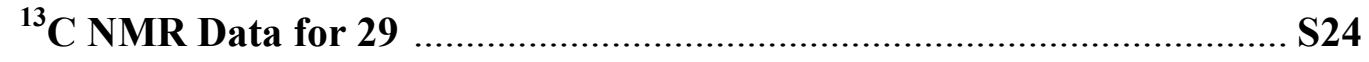


General Methods: All ${ }^{1} \mathrm{H}$ and ${ }^{13} \mathrm{C}$ NMR spectra were recorded on 400 or $500 \mathrm{MHz}$ spectrometers. Chemical shifts are reported in ppm relative to the $\mathrm{CHCl}_{3}$ peak at $7.27 \mathrm{ppm}\left({ }^{1} \mathrm{H}\right)$ or $77.0 \mathrm{ppm}\left({ }^{13} \mathrm{C}\right)$. Silica Gel $60 \mathrm{~F}_{254}$ precoated plates were used for thin layer chromatography. Flash silica gel, $60 \AA$, 230-400 mesh, $(32-63 \mu \mathrm{m})$ was used for all column chromatography. Unless otherwise specified, all reactions were run under an inert atmosphere of nitrogen. All solvents were freshly distilled before use. Yields reported refer to isolated material that was determined to be pure by NMR spectroscopy and thin layer chromatography (TLC), unless specified otherwise in the text. HPLC was performed using an OD column; 95:5 hexane:isopropanol; $1.0 \mathrm{~mL} / \mathrm{min}, 254 \mathrm{~nm}$.

$\left(1 R^{*}, 2 S^{*}\right)$-1-(2-bromo-3,4,5-trimethoxyphenyl)-2,3-dimethylbut-3-en-1-ol $\quad$ (rac-20). A mixture of indium powder $(1.0 \mathrm{~g}, 8.7 \mathrm{mmol})$ and tiglyl bromide $(2.59 \mathrm{~g}, 17.4 \mathrm{mmol})$ was stirred in anhydrous DMF $(10 \mathrm{~mL})$ for $2.5 \mathrm{~h}$ at room temperature. Tetrahydrofuran $(2.0 \mathrm{~mL})$ was added and the reaction mixture was cooled to $-78{ }^{\circ} \mathrm{C}$. A solution of 2-bromo-3,4,5trimethoxybenzaldehyde $(\mathbf{1 6})(1.20 \mathrm{~g}, 4.36 \mathrm{mmol})$ in $\mathrm{THF}(3.0 \mathrm{~mL})$ at $-78{ }^{\circ} \mathrm{C}$ was added dropwise over 10 min using a syringe pump, and the reaction mixture was stirred for $2 \mathrm{~h}$ at this temperature. The reaction was quenched by the addition of water $(3 \mathrm{~mL})$ and was diluted with ether $(100 \mathrm{~mL})$. The organic layer was washed with water $(3 \times 100 \mathrm{~mL})$ and saturated aqueous $\mathrm{NaCl}(3 \times 100 \mathrm{~mL})$, and was dried $\left(\mathrm{Na}_{2} \mathrm{SO}_{4}\right)$, filtered, and concentrated to afford 20 as a colorless solid (1.5 g, 95\%): ${ }^{1} \mathrm{H}$ NMR $\left(\mathrm{CDCl}_{3}, 500 \mathrm{MHz}\right) \delta 6.98(\mathrm{~s}, 1 \mathrm{H}), 5.13$ (d, 1H, J=1.6 Hz), 4.98 (t, $1 \mathrm{H}, J=1.6 \mathrm{~Hz}$ ), 4.90 (s, 1H), 3.90 (s, 3H), 3.89 (s, 6H), 2.74 (dq, 1H, $J=6.8,3.2 \mathrm{~Hz}), 1.97$ (d, $1 \mathrm{H}, J=1.6 \mathrm{~Hz}), 1.95$ (s, 3H), $0.91(\mathrm{dd}, 3 \mathrm{H}, J=6.8,4 \mathrm{~Hz}) ;{ }^{13} \mathrm{C} \mathrm{NMR}\left(\mathrm{CDCl}_{3}, 100 \mathrm{MHz}\right) \delta 152.5$, $150.5,148.1,136.9,111.73,107.9,107.3,72.4,61.1,61.0,56.1,43.8,22.3,11.3$; IR (film) $v_{\max }$ 3472, 2969, 2937, 1642, 1568, 1481, 1448, 1426, 1394, 1324, 1194, 1162, 1105, 1037, 1009, $893,817,738 \mathrm{~cm}^{-1}$; HRMS (ESI), $\mathrm{m} / z$ calcd for $\mathrm{C}_{15} \mathrm{H}_{21} \mathrm{BrO}_{4} \mathrm{Na}$ : 367.0515 ; found: 367.0512 .

(1S,2R)-1-(5-bromo-4-methoxybenzo[ $d][1,3]$ dioxol-6-yl)-2,3-dimethylbut-3-en-1-ol (24), (1S,2S)-1-(5-bromo-4-methoxybenzo[d][1,3] dioxol-6-yl)-2,3-dimethylbut-3-en-1-ol (25). A solution of (E)-2-methyl-2-butene-1-ylmagnesium bromide in THF (0.3 M, $23 \mathrm{~mL}, 6.87 \mathrm{mmol})$ was added dropwise over $15 \mathrm{~min}$ via syringe to a well-stirred solution of (-)-Ipc ${ }_{2} \mathrm{BCl}(2.42 \mathrm{~g}$, $7.56 \mathrm{mmol})$ in $\mathrm{THF}(30 \mathrm{~mL})$ at $-78{ }^{\circ} \mathrm{C}$. The temperature of reaction mixture was slowly raised to 
$25{ }^{\circ} \mathrm{C}$ over $3 \mathrm{~h}$ and was stirred at this temperature for $1 \mathrm{~h}$. The volatiles were removed by purging with argon gas and residue was dried under vacuum for $2 \mathrm{~h}$. The residue was taken in pentane $(20 \mathrm{~mL})$ under argon; stirring was discontinued after $30 \mathrm{~min}$, to permit the magnesium salts to settle. The clear supernatant was transferred into another flask using a double-ended needle through a Kramer filter. The pentane was removed by purging with argon gas and residue was dried under vacuum for $6 \mathrm{~h}$ to afford a pure diisopinocamphenylborane reagent $\mathbf{2 3}$ in nearly quantitative yield $(2.65 \mathrm{~g})$. A solution of aryl aldehyde $22(0.5 \mathrm{~g}, 1.93 \mathrm{mmol})$ in THF $(10 \mathrm{~mL})$ at $0{ }^{\circ} \mathrm{C}$ was transferred dropwise via cannula over 25 min to a stirred solution of $\mathbf{2 3}(0.75 \mathrm{~g}, 2.12$ $\mathrm{mmol}$ ) in THF at $-100{ }^{\circ} \mathrm{C}$ under argon. The reaction mixture was stirred at this temperature for 1 h, and was warmed to $-20{ }^{\circ} \mathrm{C}$ and treated with $3 \mathrm{~N} \mathrm{NaOH}(1.6 \mathrm{~mL})$ and $30 \% \mathrm{H}_{2} \mathrm{O}_{2}(3.2 \mathrm{~mL})$. The reaction mixture was refluxed for $3 \mathrm{~h}$, the volatiles were removed, and the residue was dissolved in EtOAc $(50 \mathrm{~mL})$ and washed with water and saturated aqueous $\mathrm{NaCl}$. The organic layer was dried $\left(\mathrm{Na}_{2} \mathrm{SO}_{4}\right)$, concentrated, and the mixture of isomers $\mathbf{2 4}$ and $\mathbf{2 5}$ were separated by flash chromatography (silica, 10\% EtOAc/hexane) to afford a pure syn and anti isomers ( $0.62 \mathrm{~g}, 97 \%$ combined yield). Syn isomer (24): 95:5 enantiomeric ratio; ${ }^{1} \mathrm{H}$ NMR $\left(\mathrm{CDCl}_{3}, 500 \mathrm{MHz}\right) \delta 6.85$ (s, 1H), 5.97 (s, 2H), 5.10 (d, 1H, $J=3.4 \mathrm{~Hz}$ ), 4.95 (app t, 1H, $J=1.4 \mathrm{~Hz}), 4.88$ (s, 1H), 4.03 (s, 3H), 2.69 (qd, 1H, $J=3.4,7.0 \mathrm{~Hz}$ ), 1.95 (br s, 1H), 1.92 (s, 3H), 0.87 (d, 3H, $J=7.0 \mathrm{~Hz}$ ); IR (film) $v_{\max } 3442,3054,2986,1642,1474,1421,1264,1080,1046,895 \mathrm{~cm}^{-1} ;{ }^{13} \mathrm{C}$ NMR $\left(\mathrm{CDCl}_{3}\right.$, $125 \mathrm{MHz}) \delta 148.5,148.0,140.0,136.4,135.0,111.7,106.4,102.9,101.6,72.6,60.1,43.9,22.2$, 11.4; HRMS (ESI), $m / z$ calcd for $\mathrm{C}_{14} \mathrm{H}_{17} \mathrm{BrO}_{4} \mathrm{Na}$ : 351.0202; found: 351.0208. Anti isomer (25): 95:5 enantiomeric ratio; ${ }^{1} \mathrm{H}$ NMR $\left(\mathrm{CDCl}_{3}, 500 \mathrm{MHz}\right) \delta 6.76(\mathrm{~s}, 1 \mathrm{H}), 5.98(\mathrm{ABq}, 2 \mathrm{H}, J=1.5 \mathrm{~Hz}$, $\Delta v=4.6 \mathrm{~Hz}) 5.04(\mathrm{dd}, 1 \mathrm{H}, J=9.1,2.5 \mathrm{~Hz}), 4.97(\operatorname{app~t}, 1 \mathrm{H}, J=1.8 \mathrm{~Hz}), 4.96(\mathrm{~s}, 1 \mathrm{H}), 4.03(\mathrm{~s}$, 3H), 2.47 (qd, 1H, $J=8.0,7.1 \mathrm{~Hz}), 2.23(\mathrm{~d}, 1 \mathrm{H}, J=2.5 \mathrm{~Hz}) 1.79$ (s, 3H), 0.91 (d, 3H $J=7.1$ $\mathrm{Hz}) ;{ }^{13} \mathrm{C}$ NMR $\left(\mathrm{CDCl}_{3}, 125 \mathrm{MHz}\right) \delta 149.1,147.2,139.7,137.0,136.2,114.2,109.0,102.2$, 101.7, 74.0, 60.2, 50.4, 19.0, 15.5; IR (film) $v_{\max } 3448,2969,1642,1474,1402,1272,1210$, 1082, 1048, 959, $895 \mathrm{~cm}^{-1}$; HRMS (ESI), $\mathrm{m} / z$ calcd for $\mathrm{C}_{14} \mathrm{H}_{17} \mathrm{BrO}_{4} \mathrm{Na}$ : 351.0208; found: 351.0195.

(Z)-trichloro(2-methylbut-2-en-1-yl)silane (26). Trichlorosilane $(6.0 \mathrm{~mL}, 60 \mathrm{mmol})$, isoprene (5.9 mL, $60 \mathrm{mmol})$, and $\mathrm{Pd}\left(\mathrm{PPh}_{3}\right)_{4}(0.1 \mathrm{~g}, 0.10 \mathrm{mmol})$ were transferred in to a sealed tube under nitrogen atmosphere and the reaction mixture was allowed to stir at $25^{\circ} \mathrm{C}$ for $15 \mathrm{~h}$. Distillation 
afforded 26 (11.4 g, 92\%) as a colorless liquid: ${ }^{1} \mathrm{H}$ NMR $\left(250 \mathrm{MHz}, \mathrm{CDCl}_{3}\right) \delta 5.46(\mathrm{q}, 1 \mathrm{H}, J=$ $6.8 \mathrm{~Hz}), 2.29(\mathrm{~s}, 2 \mathrm{H}), 1.86(\mathrm{~s}, 3 \mathrm{H}), 1.65(\mathrm{~d}, 3 \mathrm{H}, J=6.8 \mathrm{~Hz}) ;{ }^{13} \mathrm{C} \mathrm{NMR}\left(100 \mathrm{MHz}, \mathrm{CDCl}_{3}\right) \delta$ $126.4,122.6,29.7,25.4,14.3$.

(E)-trichloro(2-methylbut-2-en-1-yl)silane (27). A solution of trichlorosilane (12.0 g, 88.6 $\mathrm{mmol})$ and $\mathrm{CuCl}(0.56 \mathrm{~g}, 5.7 \mathrm{mmol})$ in $\mathrm{Et}_{2} \mathrm{O}(10 \mathrm{~mL})$ was added dropwise to a solution of tri- $n$ butylamine $(11.7 \mathrm{~g}, 63 \mathrm{mmol})$ in $\mathrm{Et}_{2} \mathrm{O}(5 \mathrm{~mL})$ at $0{ }^{\circ} \mathrm{C}$ followed by the addition of a solution of tiglylchloride $(6.0 \mathrm{~g}, 57 \mathrm{mmol})$ in $\mathrm{Et}_{2} \mathrm{O}(10 \mathrm{~mL})$. The reaction mixture was gradually warmed to $25^{\circ} \mathrm{C}$ and was allowed to stir for $12 \mathrm{~h}$ at this temperature. The solvent was removed by distillation under an argon atmosphere and pentane $(50 \mathrm{~mL})$ was added to the residue. The slurry was stirred vigorously for $4 \mathrm{~h}$ at $25^{\circ} \mathrm{C}$ and the dark pentane layer was transferred via cannula to another flask. The pentane solution was distilled at atmospheric pressure under argon to remove high boiling impurities at $160-180{ }^{\circ} \mathrm{C}$. The pot residue was subjected to fractional distillation using a Vigroux column under vacuum $\left(120-130{ }^{\circ} \mathrm{C}, c a .30 \mathrm{~mm} \mathrm{Hg}\right)$ ) to afford pure trichlorosilane $27(8.8 \mathrm{~g}, 76 \%)$ as a colorless liquid: ${ }^{1} \mathrm{H} \mathrm{NMR}\left(\mathrm{CDCl}_{3}, 500 \mathrm{MHz}\right) \delta 5.36(\mathrm{q}, 1 \mathrm{H}, J$ $=13.4 \mathrm{~Hz}), 2.32(\mathrm{~d}, 2 \mathrm{H}, J=0.66 \mathrm{~Hz}), 1.76(\mathrm{~s}, 3 \mathrm{H}), 1.64(\mathrm{~d}, 3 \mathrm{H}, J=6.7 \mathrm{~Hz}) ;{ }^{13} \mathrm{C} \mathrm{NMR}\left(\mathrm{CDCl}_{3}\right.$, $125 \mathrm{MHz}) \delta 126.2,123.5,36.1,17.9,13.8$.

\section{(3aS,7aS)-1,3-bis(4-bromobenzyl)-2-chloro-2-((Z)-2-methylbut-2-enyl)octahydro-1H-}

benzo $[\boldsymbol{d}]$-1,3,2-diazasilole (28). Diazabicycloundecane $(4.7 \mathrm{~mL}, 30.4 \mathrm{mmol})$ was added dropwise to a cooled solution of $26(2.6 \mathrm{~mL}, 15.2 \mathrm{mmol})$ in $\mathrm{CH}_{2} \mathrm{Cl}_{2}(26 \mathrm{~mL})$ at $0{ }^{\circ} \mathrm{C}$ over $10 \mathrm{~min}$. A solution of $(S, S)-N, N^{\prime}$-bis-(4-bromobenzyl)cyclohexane-1,2-diamine (6.90 g, $\left.15.2 \mathrm{mmol}\right)$ in $\mathrm{CH}_{2} \mathrm{Cl}_{2}(13 \mathrm{~mL})$ was added to the reaction mixture over $1 \mathrm{~h}$ at this temperature, which was gradually warmed to room temperature and allowed to stir for $12 \mathrm{~h}$. The solvent was removed in vacuo to obtain a pale yellow solid. The residue was diluted with pentane $(60 \mathrm{~mL})$ and stirred vigorously for $12 \mathrm{~h}$ to ensure complete precipitation of DBU salts. The suspension was filtered and the residue washed with pentane $(15 \mathrm{~mL})$ and the filtrate was concentrated under a stream of argon to afford $28(6.9 \mathrm{~g}, 78 \%)$ as a pale yellow syrup: ${ }^{1} \mathrm{H}$ NMR $\left(500 \mathrm{MHz}, \mathrm{CDCl}_{3}\right) \delta 7.41(\mathrm{~m}$, $4 \mathrm{H}), 7.29(\mathrm{~m}, 4 \mathrm{H}), 5.16(\mathrm{q}, 1 \mathrm{H}, J=5.6 \mathrm{~Hz}), 4.21(\mathrm{~d}, 1 \mathrm{H}, J=16.3 \mathrm{~Hz}), 4.06(\mathrm{~d}, 1 \mathrm{H}, J=15.6 \mathrm{~Hz})$, $3.86(\mathrm{~d}, 1 \mathrm{H}, J=15.6 \mathrm{~Hz}), 3.82(\mathrm{~d}, 1 \mathrm{H}, J=16.3 \mathrm{~Hz}), 2.81(\mathrm{~m}, 1 \mathrm{H}), 2.72(\mathrm{~m}, 1 \mathrm{H}), 1.86(\mathrm{~d}, 2 \mathrm{H}, J=$ $9.8 \mathrm{~Hz}), 1.74(\mathrm{~s}, 3 \mathrm{H}), 1.62(\mathrm{~m}, 4 \mathrm{H}), 1.48(\mathrm{~d}, 3 \mathrm{H}, \mathrm{J}=5.6 \mathrm{~Hz}) 1.22-0.89(\mathrm{~m}, 4 \mathrm{H}) ;{ }^{13} \mathrm{C}$ NMR $(125$ 
$\left.\mathrm{MHz}, \mathrm{CDCl}_{3}\right) \delta 141.6,140.2,131.1,129.9,129.6,128.9,120.2,119.4,66.3,65.4,48.0,47.3$, 31.0, 30.6, 25.8, 24.6, 23.2, 14.0; IR (film) $v_{\max } 3025$, 2937, 2861, 1591, 1486, 1442, 1405, 1342, $1278,1250,1206,1142,1105,1070,973,816 \mathrm{~cm}^{-1}$.

\section{(3aR,7aR)-1,3-bis(4-bromobenzyl)-2-chloro-2-((E)-2-methylbut-2-enyl)octahydro-1H-}

benzo[d]-1,3,2-diazasilole (29). Following the procedure for the preparation of 28, DBU (4.70

$\mathrm{mL}, 30.4 \mathrm{mmol})$ and $(R, R)-N, N^{\prime}$-bis-(4-bromobenzyl)cyclohexane-1,2-diamine (6.90 g, 15.2 mmol) were added dropwise to a cooled solution of $27(2.6 \mathrm{~mL}, 15.2 \mathrm{mmol})$ in $\mathrm{CH}_{2} \mathrm{Cl}_{2}(26 \mathrm{~mL})$ to afford $29(6.1 \mathrm{~g}, 69 \%)$ as a pale yellow syrup: ${ }^{1} \mathrm{H}$ NMR $\left(400 \mathrm{MHz}, \mathrm{CDCl}_{3}\right) \delta 7.42(\mathrm{~m}, 4 \mathrm{H})$, $7.35(\mathrm{~m}, 4 \mathrm{H}), 5.11(\mathrm{q}, 1 \mathrm{H}, J=6.4 \mathrm{~Hz}), 4.21(\mathrm{~d}, 1 \mathrm{H}, J=16.0 \mathrm{~Hz}), 4.06(\mathrm{~d}, 1 \mathrm{H}, J=15.2 \mathrm{~Hz}), 3.83$ $(\mathrm{d}, 1 \mathrm{H}, J=15.2 \mathrm{~Hz}), 3.82(\mathrm{~d}, 1 \mathrm{H}, J=16.0 \mathrm{~Hz}), 2.75(\mathrm{~m}, 2 \mathrm{H}), 1.90-1.60(\mathrm{~m}, 10 \mathrm{H}), 1.52(\mathrm{~d}, 3 \mathrm{H}, J$ $=6.4 \mathrm{~Hz}), 1.40-1.10(\mathrm{~m}, 3 \mathrm{H}) .{ }^{13} \mathrm{C} \mathrm{NMR}\left(100 \mathrm{MHz}, \mathrm{CDCl}_{3}\right) \delta$ 141.5, 140.3, 131.1, 131.0, 129.8, 129.0, 120.2, 120.1, 66.3, 65.4, 30.9, 30.6, 29.9, 24.6, 24.7, 18.1, 13.6; IR (film) $v_{\max } 3047,2983$, 2837, 2861, 1591, 1486, 1443, 1405, 1344, 1278, 1250, 1208, 1174, 1105, 1070, 1010, 973, 835 $\mathrm{cm}^{-1}$.

\section{(1S,2S)-1-(5-bromo-4-methoxybenzo[d][1,3]dioxol-6-yl)-2,3-dimethylbut-3-enyloxy)(tert-}

butyl)dimethylsilane (30). 2,6-Lutidine $(0.25 \mathrm{~mL}, 2.16 \mathrm{mmol})$ was added to a solution of alcohol $25(0.48 \mathrm{~g}, 1.45 \mathrm{mmol})$ in $\mathrm{CH}_{2} \mathrm{Cl}_{2}(10 \mathrm{~mL})$ at $-78{ }^{\circ} \mathrm{C}$. After $10 \mathrm{~min}, t-\mathrm{BuMe}_{2} \mathrm{SiOTf}(0.37$ $\mathrm{mL}, 1.56 \mathrm{mmol}$ ) was added and the reaction mixture was stirred for $20 \mathrm{~min}$ at this temperature. The reaction mixture was diluted with $\mathrm{CH}_{2} \mathrm{Cl}_{2}(50 \mathrm{~mL})$ and the organic layer was washed with water and saturated aqueous $\mathrm{NH}_{4} \mathrm{Cl}$. The organic layer was dried $\left(\mathrm{Na}_{2} \mathrm{SO}_{4}\right)$, concentrated, and the residue was purified by flash chromatography (5\% EtOAc/hexane) to afford $\mathbf{3 0}(0.63 \mathrm{~g}, 98 \%)$ as a syrup: ${ }^{1} \mathrm{H}$ NMR $\left(\mathrm{CDCl}_{3}, 500 \mathrm{MHz}\right) \delta 6.71(\mathrm{~s}, 1 \mathrm{H}), 5.97(\mathrm{ABq}, 2 \mathrm{H}, J=2.0 \mathrm{~Hz}, \Delta v=10.7 \mathrm{~Hz})$, $5.02(\mathrm{~d}, 1 \mathrm{H}, J=6.9 \mathrm{~Hz}), 4.75$ (s, 1H), $4.66(\mathrm{~s}, 1 \mathrm{H}), 4.03$ (s, 3H), 2.42 (app sextet, 1H, $J=5.3,6.7$ Hz), 1.73 (s, 3H), 0.91 (d, 3H, J=7.2 Hz), 0.85 (s, 9H), 0.03 (s, 3H), -0.24 (s, 3H); ${ }^{13} \mathrm{C}$ NMR $\left(\mathrm{CDCl}_{3}, 125 \mathrm{MHz}\right) \delta 148.5,147.0,139.2,138.3,136.2,112.2,107.3,103.0,76.2,60.1,49.2$, 25.8, 20.6, 18.0, 16.2, -4.7, -5.2; IR (film) $v_{\max } 3685,2957,2856,1607,1472,1447,1402,1375$, $1261,1210,1126,1080,1047,961,895 \mathrm{~cm}^{-1}$; HRMS (ESI), $\mathrm{m} / z$ calcd for $\mathrm{C}_{20} \mathrm{H}_{31} \mathrm{BrO}_{4} \mathrm{SiNa}$ : 465.1072; found: 465.1066 . 


\section{((1S,2S,3S)-4-(4-(benzyloxy)benzo[d][1,3]dioxol-6-yl)-1-(5-bromo-4-methoxy-}

benzo $[d][1,3]$ dioxol-6-yl)-2,3-dimethylbutoxy)(tert-butyl)dimethylsilane (35). A solution of $30(0.25 \mathrm{~g}, 0.56 \mathrm{mmol})$ in THF $(2 \mathrm{~mL})$ was cooled to $0{ }^{\circ} \mathrm{C}$, and a solution of 9 -BBN in THF $(0.50$ M, $1.42 \mathrm{~mL}, 0.81 \mathrm{mmol}$ ) was added dropwise by syringe over $25 \mathrm{~min}$ at this temperature. The reaction mixture was allowed to warm to $25^{\circ} \mathrm{C}$ over $4 \mathrm{~h}$, and was stirred at this temperature for 6 $\mathrm{h}$, when it was transferred dropwise to a flask containing a cooled solution of aryl bromide $\mathbf{3 3}$ (0.38 g, $1.23 \mathrm{mmol}), \mathrm{Pd}\left(\mathrm{PPh}_{3}\right)_{4}(39 \mathrm{mg}, 0.06 \mathrm{mmol})$ and aqueous $\mathrm{NaOH}(4 \mathrm{M}, 0.46 \mathrm{~mL}, 1.84$ $\mathrm{mmol})$ in THF $(2 \mathrm{~mL})$ at $0{ }^{\circ} \mathrm{C}$. The reaction mixture was warmed at $70{ }^{\circ} \mathrm{C}$ for $22 \mathrm{~h}$ and was allowed to cool to $25^{\circ} \mathrm{C}$. The volatiles were removed, and the residue was diluted with $\mathrm{CH}_{2} \mathrm{Cl}_{2}$ $(20 \mathrm{~mL})$, passed through bed of Celite. The filtrate was washed with water and saturated aqueous $\mathrm{NaCl}$, and the organic layer was dried $\left(\mathrm{Na}_{2} \mathrm{SO}_{4}\right)$, concentrated, and the residue was purified by flash chromatography (5-10\% EtOAc/hexane) to afford $35(0.29 \mathrm{~g}, 77 \%)$ as a syrup: ${ }^{1} \mathrm{H}$ NMR $\left(\mathrm{CDCl}_{3}, 500 \mathrm{MHz}\right) \delta 7.44(\mathrm{~d}, 2 \mathrm{H}, J=7.3 \mathrm{~Hz}), 7.38(2 \mathrm{~d}, 2 \mathrm{H}, J=7.7 \mathrm{~Hz}), 7.32(2 \mathrm{~d}, 1 \mathrm{H}, J=7.3$ Hz), $6.79(\mathrm{~s}, 1 \mathrm{H}), 6.42(\operatorname{app~s}, 1 \mathrm{H}), 6.40(\mathrm{~d}, 1 \mathrm{H}, J=0.9 \mathrm{~Hz}), 5.96(\mathrm{ABq}, 2 \mathrm{H}, J=1.4 \mathrm{~Hz}, \Delta v=$ $20.5 \mathrm{~Hz}), 5.94$ (s, 2H), 5.17 (s, 2H), 5.09 (d, 1H, J = $7.7 \mathrm{~Hz}), 4.04$ (s, 3H), 2.96 (dd, 1H, J = 13.2, $3.0 \mathrm{~Hz}), 2.30-2.15(\mathrm{~m}, 1 \mathrm{H}), 2.08(\mathrm{dd}, 1 \mathrm{H}, J=13.1,11.8 \mathrm{~Hz}), 1.80(\mathrm{~m}, 1 \mathrm{H}), 0.90$ (s, 9H), 0.75 (d, $3 \mathrm{H}, J=6.8 \mathrm{~Hz}), 0.72(\mathrm{~d}, 3 \mathrm{H}, J=7.2 \mathrm{~Hz}), 0.12(\mathrm{~s}, 3 \mathrm{H})-0.26(\mathrm{~s}, 3 \mathrm{H}) ;{ }^{13} \mathrm{C} \mathrm{NMR}\left(\mathrm{CDCl}_{3}, 125\right.$ MHz) $\delta 148.8$ (2 C), 142.3, 139.5, 138.8, 137.0, 136.7, 136.3, 133.5, 128.5, 128.0, 127.6, 110.3, 107.7, 103.5, 102.9, 101.6, 101.1, 76.0, 71.4, 60.1, 46.3, 37.2, 34.0, 26.0, 18.2, 18.1, 10.5 -4.5, 4.7; IR (film) $v_{\max } 2952,2860,1632,1604,1508,1472,1405,1374,1255,1083,1046,960,934$, 854, 837, 777, 736, $\mathrm{cm}^{-1}$; HRMS (ESI), $\mathrm{m} / \mathrm{z}$ calcd for $\mathrm{C}_{34} \mathrm{H}_{43} \mathrm{BrO}_{7} \mathrm{SiNa}$ : 693.1859; found: 693.1849.

\section{$((1 S, 2 S, 3 S)-4-(4-h y d r o x y b e n z o[d][1,3]$ dioxol-6-yl)-1-(5-bromo-4-methoxybenzo[d][1,3]di-} oxol-6-yl)-2,3-dimethylbutoxy)(tert-butyl)dimethylsilane (36). A solution of diarylbutane 35 $(0.20 \mathrm{~g}, 0.29 \mathrm{mmol})$ and $5 \% \mathrm{Pd} / \mathrm{C}(15 \mathrm{mg})$ in EtOAc $(5 \mathrm{~mL})$ was vigorously stirred under a hydrogen atmosphere (balloon) at room temperature for $6 \mathrm{~h}$. The reaction mixture was filtered, the filtrate was concentrated, and the residue was purified by flash chromatography (20\% EtOAc/hexane) to afford $36(0.17 \mathrm{~g}, 98 \%)$ as a syrup: ${ }^{1} \mathrm{H} \mathrm{NMR}\left(\mathrm{CDCl}_{3}, 500 \mathrm{MHz}\right) \delta 6.78(\mathrm{~s}$, $1 \mathrm{H}), 6.35(\mathrm{~d}, 1 \mathrm{H}, J=1.2 \mathrm{~Hz}), 6.33(\operatorname{app~s}, 1 \mathrm{H}), 5.98(\mathrm{ABq}, 2 \mathrm{H}, J=1.4 \mathrm{~Hz}, \Delta v=12.2 \mathrm{~Hz}), 5.93$ (s, 2H), 5.09 (d, 1H, J = 7.8 Hz), 4.75 (br s, 1H), 4.03 (s, 3H), 2.93 (dd, 1H, J = 13.2, 3.2 Hz), 
2.35-2.15 (m, 1H), $2.08(\mathrm{dd}, 1 \mathrm{H}, J=13.2,11.8 \mathrm{~Hz}), 1.90-1.75(\mathrm{~m}, 1 \mathrm{H}), 0.91(\mathrm{~s}, 9 \mathrm{H}), 0.78(\mathrm{~d}, 3 \mathrm{H}$, $J=6.9 \mathrm{~Hz}), 0.71(\mathrm{~d}, 3 \mathrm{H}, J=7.2 \mathrm{~Hz}), 0.10(\mathrm{~s}, 3 \mathrm{H})-0.27(\mathrm{~s}, 3 \mathrm{H}) ;{ }^{13} \mathrm{C} \mathrm{NMR}\left(\mathrm{CDCl}_{3}, 125 \mathrm{MHz}\right) \delta$ $148.8,148.6,139.4,138.8,138.7,137.0,136.3$, 131.9, 111.3, 107.8, 102.9, 102.7, 101.6, 101.2, $75.9,60.2,46.5,37.1,34.0,26.0,18.3,18.1,10.5-4.5,-4.7$; IR (neat) $v_{\max } 2956,2857,1634$, 1608, 1506, 1474, 1372, 1277, 1256, 1191, 1125, 1045, 960, 857, 834, 776, $736 \mathrm{~cm}^{-1}$; HRMS (ESI), $m / z$ calcd for $\mathrm{C}_{27} \mathrm{H}_{37} \mathrm{BrO}_{7} \mathrm{SiNa}$ : 603.1390; found: 603.1383 .

\section{((1S,2S,3S)-4-(5-bromo-4-hydroxybenzo[d][1,3]dioxol-6-yl)-1-(5-bromo-4-methoxy-}

benzo $[d][1,3]$ dioxol-6-yl)-2,3-dimethylbutoxy)(tert-butyl)dimethylsilane (37). A solution of 1,4-diarylbutane $36(0.15 \mathrm{~g}, 0.25 \mathrm{mmol})$ in dioxane $(5 \mathrm{~mL})$ was cooled to $15^{\circ} \mathrm{C}$. A solution of $N$ bromosuccinimide (46 mg, $0.26 \mathrm{mmol})$ in dioxane $(3 \mathrm{~mL})$ was added dropwise over $1 \mathrm{~h}$ at this temperature. The reaction mixture was stirred for $11 \mathrm{~h}$ between $15-19{ }^{\circ} \mathrm{C}$ and was quenched by the addition of saturated aqueous $\mathrm{Na}_{2} \mathrm{~S}_{2} \mathrm{O}_{3}(1 \mathrm{~mL})$. The mixture was concentrated, and the residue was dissolved in EtOAc $(25 \mathrm{~mL})$ and washed with saturated aqueous $\mathrm{Na}_{2} \mathrm{~S}_{2} \mathrm{O}_{3}$, water, and saturated aqueous $\mathrm{NaCl}$. The organic layer was dried $\left(\mathrm{Na}_{2} \mathrm{SO}_{4}\right)$, concentrated, and the residue was purified by flash chromatography (silica, 15\% EtOAc/hexane) to afford $37(0.15 \mathrm{~g}, 90 \%)$ as a syrup: ${ }^{1} \mathrm{H} \mathrm{NMR}\left(\mathrm{CDCl}_{3}, 500 \mathrm{MHz}\right) \delta 6.80(\mathrm{~s}, 1 \mathrm{H}), 6.46(\mathrm{~s}, 1 \mathrm{H}), 5.99(\mathrm{~s}, 2 \mathrm{H}), 5.98(\mathrm{ABq}, 2 \mathrm{H}, J$ $=1.4 \mathrm{~Hz}, \Delta v=13.6 \mathrm{~Hz}), 5.52(\mathrm{~s}, 1 \mathrm{H}), 5.09(\mathrm{~d}, 1 \mathrm{H}, J=7.5 \mathrm{~Hz}), 4.04(\mathrm{~s}, 3 \mathrm{H}), 2.94(\mathrm{dd}, 1 \mathrm{H}, J=$ 13.9, 4.2 Hz), 2.59 (dd, 1H, $J=13.9,11.1 \mathrm{~Hz}), 2.30-2.15(\mathrm{~m}, 1 \mathrm{H}), 1.90-1.75(\mathrm{~m}, 1 \mathrm{H}), 0.89$ (s, $9 \mathrm{H}), 0.81(\mathrm{~d}, 3 \mathrm{H}, J=7.0 \mathrm{~Hz}), 0.79(\mathrm{~d}, 3 \mathrm{H}, J=7.2 \mathrm{~Hz}), 0.10(\mathrm{~s}, 3 \mathrm{H}), 0.27(\mathrm{~s}, 3 \mathrm{H}) ;{ }^{13} \mathrm{C}$ NMR $\left(\mathrm{CDCl}_{3}, 125 \mathrm{MHz}\right) \delta 148.7,148.2,139.5,138.6,136.3$ (2 C), 134.9, 132.4, 107.7, 106.6, 103.0, $102.9,101.9,101.6,76.1,60.1,46.1,37.3,33.7,26.0,18.0,17.7,11.4,-4.5,-4.8$; IR (neat) $v_{\max }$ 2956, 2886, 2355, 1630, 1605, 1476, 1275, 1251, 1188, 1125, 1084, 959, 932, 837, 776, $735 \mathrm{~cm}^{-}$ ${ }^{1}$; HRMS (ESI), $m / z$ calcd for $\mathrm{C}_{27} \mathrm{H}_{36} \mathrm{Br}_{2} \mathrm{O}_{7} \mathrm{SiNa}$ : 681.0495; found: 681.0515 .

\section{$((1 S, 2 S, 3 S)-1,4-b i s(5-b r o m o-4-m e t h o x y b e n z o[d][1,3]$ dioxol-6-yl)-2,3-dimethylbutoxy)(tert-}

butyl)dimethylsilane (38). A mixture of 37 (100 mg, $0.15 \mathrm{mmol}), \mathrm{K}_{2} \mathrm{CO}_{3}(25 \mathrm{mg}, 0.22 \mathrm{mmol})$ and dimethyl sulfate $(15 \mu \mathrm{L}, 0.18 \mathrm{mmol})$ in acetone $(5 \mathrm{~mL})$ was warmed at $56^{\circ} \mathrm{C}$ for $2 \mathrm{~h}$. After cooling to room temperature, the mixture was filtered and concentrated. The residue was dissolved in EtOAc $(50 \mathrm{~mL})$, and was washed with water and saturated aqueous $\mathrm{NaCl}$. The organic layer was dried $\left(\mathrm{Na}_{2} \mathrm{SO}_{4}\right)$, concentrated, and the residue was purified by flash 
chromatography (silica, $15 \%$ EtOAc/hexane) to afford $38(0.096 \mathrm{~g}, 95 \%)$ as colorless solid: ${ }^{1} \mathrm{H}$ NMR $\left(\mathrm{CDCl}_{3}, 500 \mathrm{MHz}\right) \delta 6.80(\mathrm{~s}, 1 \mathrm{H}), 6.53(\mathrm{~s}, 1 \mathrm{H}), 5.98(\mathrm{ABq}, 2 \mathrm{H}, J=1.4 \mathrm{~Hz}, \Delta v=14.6 \mathrm{~Hz})$, 5.95 (s, 2H), $5.10(\mathrm{~d}, 1 \mathrm{H}, J=7.6 \mathrm{~Hz}), 4.03(\mathrm{~s}, 3 \mathrm{H}), 4.02(\mathrm{~s}, 3 \mathrm{H}), 2.92(\mathrm{dd}, 1 \mathrm{H}, J=13.8,4.2 \mathrm{~Hz})$, $2.67(\mathrm{dd}, 1 \mathrm{H}, J=13.8,11.0 \mathrm{~Hz}), 2.35-2.20(\mathrm{~m}, 1 \mathrm{H}), 1.90-1.80(\mathrm{~m}, 1 \mathrm{H}), 0.89$ (s, 9H), 0.82 (d, 3H, $J=7.0 \mathrm{~Hz}), 0.78(\mathrm{~d}, 3 \mathrm{H}, J=7.2 \mathrm{~Hz}), 0.10(\mathrm{~s}, 3 \mathrm{H}),-0.27(\mathrm{~s}, 3 \mathrm{H}) ;{ }^{13} \mathrm{C} \mathrm{NMR}\left(\mathrm{CDCl}_{3}, 125 \mathrm{MHz}\right) \delta$ 148.8, 148.3, 140.3, 139.5, 138.7, 136.4, 135.8, 135.5, 110.0, 107.7, 104.6, 103.0, 101.6, 101.4, 76.1, 60.1, 60.0, 46.3 37.3, 33.8, 26.0, 18.1, 17.7, 11.5, -4.5, -4.7; IR (film) $v_{\max } 2956,2884$, 2856, 1606, 1499, 1473, 1447, 1398, 1373, 1277, 1211, 1085, 1049, 1004, 960, 935, 856, 836, 776, $674 \mathrm{~cm}^{-1}$; HRMS (ESI), $m / z$ calcd for $\mathrm{C}_{28} \mathrm{H}_{38} \mathrm{Br}_{2} \mathrm{O}_{7} \mathrm{SiNa}$ : 697.0634; found: 697.0631.

\section{(1S,2S)-(1-(2-Bromo-3,4,5-trimethoxyphenyl)-2,3-dimethylbut-3-enyloxy)(tert-}

butyl)dimethylsilane (39). Following the procedure for the preparation of 30, 2,6-lutidine $(0.25$ $\mathrm{mL}, 2.16 \mathrm{mmol})$ and $t$-BuMe $\mathrm{SiOTf}(0.37 \mathrm{~mL}, 1.56 \mathrm{mmol})$ were added to a solution of alcohol $21(0.48 \mathrm{~g}, 1.45 \mathrm{mmol})$ in $\mathrm{CH}_{2} \mathrm{Cl}_{2}(10 \mathrm{~mL})$ at $-78{ }^{\circ} \mathrm{C}$. Purification afforded $39(0.63 \mathrm{~g}, 98 \%)$ as a syrup: ${ }^{1} \mathrm{H}$ NMR $\left(\mathrm{CDCl}_{3}, 400 \mathrm{MHz}\right) \delta 6.85(\mathrm{~s}, 1 \mathrm{H}), 5.03(\mathrm{~d}, 1 \mathrm{H}, J=6.8 \mathrm{~Hz}), 4.75($ app t $, 1 \mathrm{H}, J=$ $1.7 \mathrm{~Hz}$ ), 4.66 (s, 1H), 3.89 (s, 3H), 3.88 (s, 3H), 3.84 (s, 3H), 2.45 (quintet, 1H, J = 7.0, Hz), 1.72 (s, 3H), 0.95 (d, 3H, J = 7.2 Hz), 0.86 (s, 9H), 0.04 (s, 3H), -0.24 (s, 3H); ${ }^{13} \mathrm{C} \mathrm{NMR}\left(\mathrm{CDCl}_{3}, 100\right.$ MHz) $\delta$ 153.0, 150.2, 147.3, 142.4, 139.7, 112.6, 109.2, 107.8, 76.5, 61.5, 61.2, 56.3, 49.4, 26.1, 21.1, 18.4, 16.7, -4.4, -4.8; IR (film) $v_{\max } 3685,2957,2856,1607,1472,1447,1402,1375$, 1261, 1210, 1126, 1080, 1047, 961, $895 \mathrm{~cm}^{-1}$; HRMS (ESI), $\mathrm{m} / z$ calcd for $\mathrm{C}_{21} \mathrm{H}_{35} \mathrm{BrO}_{4} \mathrm{SiNa}$ : 465.1073; found: 465.1066 .

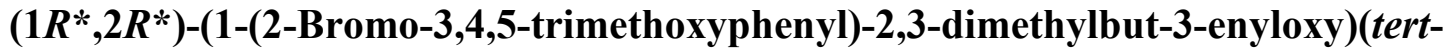

butyl)dimethylsilane (rac-39). A dispersion of $\mathrm{NaH}(1.10 \mathrm{~g}, 27.4 \mathrm{mmol})$ in THF $(10 \mathrm{~mL})$ was cooled to $-15^{\circ} \mathrm{C}$. A solution of $\mathrm{rac}-\mathbf{2 0}(3.15 \mathrm{~g}, 9.10 \mathrm{mmol})$ in THF $(20 \mathrm{~mL})$ was added dropwise over $10 \mathrm{~min}$ and the resulting slurry was stirred at $-25{ }^{\circ} \mathrm{C}$ for $1 \mathrm{~h}$. A solution of tertbutyldimethylsilyl chloride $(2.75 \mathrm{~g}, 18.2 \mathrm{mmol})$ in THF $(25 \mathrm{~mL})$ was added to the slurry at -15 ${ }^{\circ} \mathrm{C}$ over 20 min using a syringe pump. The reaction mixture was allowed to warm to room temperature, and was stirred for $24 \mathrm{~h}$. Excess $\mathrm{NaH}$ was quenched by the addition of water at 0 ${ }^{\circ} \mathrm{C}$. The reaction mixture was diluted with ether $(200 \mathrm{~mL})$ and was washed with saturated aqueous $\mathrm{NaCl}(4 \times 150 \mathrm{~mL})$. The organic layer was dried $\left(\mathrm{Na}_{2} \mathrm{SO}_{4}\right)$, filtered, concentrated, and the residue was purified by flash chromatography (silica gel, 10\% EtOAc/hexane) to afford 39 
(4.0 g, 95\%) as a colorless solid: ${ }^{1} \mathrm{H}$ NMR $\left(400 \mathrm{MHz}, \mathrm{CDCl}_{3}\right) \delta 6.94(\mathrm{~s}, 1 \mathrm{H}), 5.07(\mathrm{~d}, 1 \mathrm{H}, J=3.2$ Hz), 4.82 (s, 1H), 4.77 (s, 1H), 3.95 (s, 3H), 3.94 (s, 3H), 3.89 (s, 3H), 2.43 (m, 1H), 1.86 (s, $3 \mathrm{H}), 0.97$ (d, 3H, $J=6.8 \mathrm{~Hz}), 0.90$ (s, 9H), 0.03 (s, 3H), -0.21 (s, 3H); ${ }^{13} \mathrm{C}$ NMR (100 MHz, $\left.\mathrm{CDCl}_{3}\right) \delta 152.1,150.0,147.2,141.8,139.2,111.8,107.9,107.8,74.7,61.1,61.0,55.9,45.7$, 25.9, 25.7, 22.2, 18.2, 12.0, -4.8, -5.2; IR (film) $v_{\max } 2933,1481,1254,1108,890 \mathrm{~cm}^{-1}$; HRMS (ESI), $m / z$ calcd for $\mathrm{C}_{21} \mathrm{H}_{35} \mathrm{BrO}_{4} \mathrm{SiNa}$ : 481.1386 ; found: 481.1351 .

((1S,2S,3S)-1-(2-bromo-3,4,5-trimethoxyphenyl)-4-(4-methoxybenzo[d][1,3]dioxol-6-yl)-2,3dimethylbutoxy)(tert-butyl)dimethylsilane (41). A solution of 39 (0.042 g, $0.093 \mathrm{mmol})$ in THF $(0.50 \mathrm{~mL})$ was cooled to $0{ }^{\circ} \mathrm{C}$, and a solution of $9-\mathrm{BBN}$ in THF $(0.50 \mathrm{M}, 0.32 \mathrm{~mL}, 0.16$ mmol) was added dropwise over $10 \mathrm{~min}$. The reaction mixture was allowed to warm to $10{ }^{\circ} \mathrm{C}$ over a period of $2 \mathrm{~h}$ and was stirred at this temperature for $10 \mathrm{~h}$. In another flask, aryl bromide 32 (0.032 g, $0.14 \mathrm{mmol}), \mathrm{Pd}\left(\mathrm{PPh}_{3}\right)_{4}(0.012 \mathrm{~g}, 0.01 \mathrm{mmol}), \mathrm{Ph}_{3} \mathrm{P}(0.010 \mathrm{~g}, 0.037 \mathrm{mmol})$ and $\mathrm{NaOH}$ $(0.015 \mathrm{~g}, 0.372 \mathrm{mmol})$ in wet THF $(1.0 \mathrm{~mL})$ was warmed at $40{ }^{\circ} \mathrm{C}$ for $2 \mathrm{~h}$ when a light green solution formed. This solution was cooled to $0{ }^{\circ} \mathrm{C}$ and the the solution of alkylborane $\mathbf{4 0}$ was added dropwise over a period of $10 \mathrm{~min}$. The reaction mixture was warmed at $70{ }^{\circ} \mathrm{C}$ for $28 \mathrm{~h}$, cooled to room temperature, and the volatiles were removed. The residue was diluted with $\mathrm{CH}_{2} \mathrm{Cl}_{2}(10 \mathrm{~mL})$, passed through a pad of Celite, and the filtrate was washed with water $(2 \times 10$ $\mathrm{mL})$, and saturated aqueous $\mathrm{NaCl}(2 \times 10 \mathrm{~mL})$. The organic layer was dried $\left(\mathrm{Na}_{2} \mathrm{SO}_{4}\right)$, filtered, concentrated, and the residue was purified by flash chromatography (silica gel, 5-10\% EtOAc/hexane) to afford $41(0.034 \mathrm{~g}, 61 \%)$ as a colorless oil: IR (film) $v_{\max } 2929,2856,2367$, 1458, 1426, 1395, 1253, 1196, 1106, 836, $776 \mathrm{~cm}^{-1}$; ${ }^{1} \mathrm{H}$ NMR $\left(\mathrm{CDCl}_{3}, 400 \mathrm{MHz}\right) \delta 6.92$ (s, IH), $6.41(\mathrm{~s}, 1 \mathrm{H}), 6.36(\mathrm{~s}, 1 \mathrm{H}), 5.94(\mathrm{~s}, 2 \mathrm{H}), 5.10(\mathrm{~d}, 1 \mathrm{H}, J=8.0 \mathrm{~Hz}), 3.90(\mathrm{~s}, 3 \mathrm{H}), 3.89(\mathrm{~s}, 6 \mathrm{H}), 3.88$ (s, 3H), $3.00(\mathrm{dd}, 1 \mathrm{H}, J=13.0,2.8 \mathrm{~Hz}), 2.24(\mathrm{~m}, 1 \mathrm{H}), 2.14(\mathrm{~m}, 1 \mathrm{H}), 1.85(\mathrm{~m}, 1 \mathrm{H}), 0.90(\mathrm{~s}, 9 \mathrm{H})$, $0.80(\mathrm{~d}, 3 \mathrm{H}, J=6.8 \mathrm{~Hz}, 0.87), 0.74(\mathrm{~d}, 3 \mathrm{H}, J=7.2 \mathrm{~Hz}), 0.14(\mathrm{~s}, 3 \mathrm{H}),-0.27(\mathrm{~s}, 3 \mathrm{H}) ;{ }^{13} \mathrm{C} \mathrm{NMR}$ $\left(\mathrm{CDCl}_{3}, 100 \mathrm{MHz}\right) \delta 152.7,149.9,148.6,143.3,142.1,139.9,136.8,133.1,109.5,108.1,107.3$, 103.1, 101.1, 75.8, 61.2, 61.0, 56.5, 56.0, 46.3, 37.2, 33.9, 29.7, 25.9, 18.3, 18.1, 14.1, 10.6, -4.7, -4.8; HRMS (ESI) $m / z$ calcd for $\mathrm{C}_{29} \mathrm{H}_{43} \mathrm{BrO}_{7} \mathrm{SiNa}$ : 633.1859; found: 633.1855.

((1S,2S,3S)-1-(2-Bromo-3,4,5-trimethoxyphenyl)-4-(5-bromo-4-

methoxybenzo[d][1,3]dioxol-6-yl)-2,3-dimethylbutoxy)(tert-butyl)dimethylsilane $\quad(42):$ A 
solution of NBS (11.6 mg, $0.065 \mathrm{mmol})$ in $\mathrm{CHCl}_{3}(0.7 \mathrm{~mL})$ was added to a solution of 41 (4.0 $\mathrm{mg}, 0.065 \mathrm{mmol})$ in $\mathrm{CHCl}_{3}(0.7 \mathrm{~mL})$ at $22{ }^{\circ} \mathrm{C}$ and the reaction mixture was stirred vigorously for $4.5 \mathrm{~h}$ at this temperature. The volatiles were removed, and the residue was diluted with EtOAc (5 $\mathrm{mL})$, washed with saturated aqueous $\mathrm{Na}_{2} \mathrm{~S}_{2} \mathrm{O}_{3}(10 \mathrm{~mL})$, saturated aqueous $\mathrm{NH}_{4} \mathrm{Cl}(2 \times 10 \mathrm{~mL})$, and saturated aqueous $\mathrm{NaCl}(2 \times 10 \mathrm{~mL})$. The organic layer was dried $\left(\mathrm{Na}_{2} \mathrm{SO}_{4}\right)$, filtered, concentrated, and the residue was purified by flash chromatography (silica gel, 5-10\% EtOAc/hexane) to afford 42 (4.0 mg, 89\%) as a colorless oil: IR (film) $v_{\max } 2929,2855,1606$, 1567, 1477, 1395, 1256, 1106, 1053, 836, $776 \mathrm{~cm}^{-1} ;{ }^{1} \mathrm{H}$ NMR $\left(\mathrm{CDCl}_{3}, 500 \mathrm{MHz}\right) \delta 6.94(\mathrm{~s}, 1 \mathrm{H})$, $6.56(\mathrm{~s}, 1 \mathrm{H}), 5.95$ (s, 2H), 5.09 (d, 1H, J=7.6 Hz), 4.03 (s, 3H), 3.90 (s, 3H), 3.89 (s, 3H), 3.87 (s, 3H), 2.97 (dd, 1H, $J=13.8,4.2 \mathrm{~Hz}), 2.69$ (dd, 1H, $J=13.7,10.9 \mathrm{~Hz}), 2.25$ (m, 1H), 1.89 (m, 1H), 0.84 (d, 3H, $J=7.0 \mathrm{~Hz}$ ), 0.81 (d, 3H, $J=7.3 \mathrm{~Hz}$ ), 0.90 (s, 9H), 0.11 (s, 3H), -0.29 (s, 3H); ${ }^{13} \mathrm{C}$ NMR $\left(\mathrm{CDCl}_{3}, 125 \mathrm{MHz}\right) \delta$ 152.7, 150.1, 148.3, 142.1, 140.3, 139.8, 135.8, 135.5, 109.9, 109.3, 108.7, 107.5, 104.6, 101.4, 76.1, 61.2, 61.0, 60.0, 56.0, 45.9, 37.6, 33.9, 29.7, 25.9, 18.1, 17.6, 11.9, -4.6, -4.7; HRMS (ESI), $m / z$ calcd for $\mathrm{C}_{29} \mathrm{H}_{42} \mathrm{Br}_{2} \mathrm{O}_{7} \mathrm{SiNa}$ : 711.0964; found: 711.0961 .

$\left(\left(1 R^{*}, 2 S^{*}, 3 S^{*}\right)-1-(2-b r o m o-3,4,5-t r i m e t h o x y p h e n y l)-4-(4-m e t h o x y b e n z o[d][1,3]\right.$ dioxol-6-yl)2,3-dimethylbutoxy)(tert-butyl)dimethylsilane (rac-41). A solution of 9-BBN in THF (0.50 M, $5.86 \mathrm{~mL}, 2.93 \mathrm{mmol}$ ) was added dropwise over $30 \mathrm{~min}$ via a syringe pump to a solution of rac$39(0.750 \mathrm{~g}, 1.63 \mathrm{mmol})$ in THF $(7 \mathrm{~mL})$ at $0{ }^{\circ} \mathrm{C}$. The reaction mixture was allowed to warm to 10 ${ }^{\circ} \mathrm{C}$ over a period of $2 \mathrm{~h}$ and was stirred at this temperature for $8 \mathrm{~h}$. In another flask, a mixture of aryl bromide 32 (0.381 g, $1.65 \mathrm{mmol}), \mathrm{Pd}\left(\mathrm{PPh}_{3}\right)_{4}$ (0.225 g, $\left.0.195 \mathrm{mmol}\right), \mathrm{Ph}_{3} \mathrm{P}$ (0.171 g, 0.652 $\mathrm{mmol})$ and aqueous $\mathrm{NaOH}(0.261 \mathrm{~g}$, dissolved in $0.8 \mathrm{~mL}$ water, $6.52 \mathrm{mmol})$ in THF $(8.0 \mathrm{~mL})$ was warmed at $40{ }^{\circ} \mathrm{C}$ for $2 \mathrm{~h}$ when a light green solution formed. The reaction mixture was cooled to $0{ }^{\circ} \mathrm{C}$ and the trialkylborane solution was added dropwise over a period of $10 \mathrm{~min}$. The reaction mixture was warmed at $70{ }^{\circ} \mathrm{C}$ for $32 \mathrm{~h}$, cooled to room temperature, and the volatiles were removed. The residue was diluted with $\mathrm{CH}_{2} \mathrm{Cl}_{2}(10 \mathrm{~mL})$, passed through a pad of Celite, and the filtrate was washed with water $(2 \times 40 \mathrm{~mL})$, and saturated aqueous $\mathrm{NaCl}(2 \times 40 \mathrm{~mL})$. The organic layer was dried filtered, concentrated, and the residue was purified by flash chromatography (silica gel, 5-10\% EtOAc/hexane) to afford rac-41 (0.60 g, 60\%) as a colorless solid: ${ }^{1} \mathrm{H} \mathrm{NMR}\left(\mathrm{CDCl}_{3}, 400 \mathrm{MHz}\right) \delta 6.89$ (s, 1H), 6.31 (d, 2H, J=3.2 Hz), 5.93 (s, 2H), 5.18 (d, $1 \mathrm{H}, J=4.4 \mathrm{~Hz}$ ), $3.90(\mathrm{~s}, 6 \mathrm{H}), 3.87$ (s, 3H), 3.86 (s, 3H), 2.97 (dd, 1H, J=6.0, $2.8 \mathrm{~Hz}), 2.04$ (dd, 
1H, $J=13.2,11.2 \mathrm{~Hz}), 1.69(\mathrm{~m}, 2 \mathrm{H}), 0.97$ (d, 3H, $J=6.8 \mathrm{~Hz}), 0.92(\mathrm{~s}, 9 \mathrm{H}), 0.87$ (d, 3H, $J=6.4$ $\mathrm{Hz}), 0.14(\mathrm{~s}, 3 \mathrm{H}),-0.22(\mathrm{~s}, 3 \mathrm{H}) ;{ }^{13} \mathrm{C} \mathrm{NMR}\left(\mathrm{CDCl}_{3}, 125 \mathrm{MHz}\right) \delta 152.4,150.1,148.5,143.3$, $142.1,139.6,136.5,133.1,108.5,108.1,107.9,103.0,101.0,75.1,61.1,60.9,56.4,56.0,45.6$, 41.9, 39.3, 36.9, 31.6, 18.3, 18.1, 14.2, 10.5, -4.4, -4.9; IR (film) $v_{\max } 3054,2986,1422,1264$, 896, 755, $710 \mathrm{~cm}^{-1}$; HRMS (ESI), $\mathrm{m} / z$ calcd for $\mathrm{C}_{29} \mathrm{H}_{43} \mathrm{BrO}_{7} \mathrm{SiNa}$ : 633.1859; found: 633.1867 .

\section{$\left(\left(1 R^{*}, 2 S^{*}, 3 S^{*}\right)-1-(2-\right.$ bromo-3,4,5-trimethoxyphenyl)-4-(5-bromo-4-} methoxybenzo[d][1,3] dioxol-6-yl)-2,3-dimethylbutoxy)(tert-butyl)dimethylsilane (rac-42). A solution of $N$-bromosuccinimide $(0.08 \mathrm{~g}, 0.449 \mathrm{mmol})$ in $\mathrm{CHCl}_{3}(5.0 \mathrm{~mL})$ was added to a solution of $41(0.273 \mathrm{~g}, 0.446 \mathrm{mmol})$ in $\mathrm{CHCl}_{3}(2.5 \mathrm{~mL})$ at $22{ }^{\circ} \mathrm{C}$, and the reaction mixture was stirred vigorously for $7 \mathrm{~h}$ at this temperature. The volatiles were removed and the residue was diluted with EtOAc $(10 \mathrm{~mL})$, which was washed with saturated aqueous $\mathrm{Na}_{2} \mathrm{~S}_{2} \mathrm{O}_{3}(10 \mathrm{~mL})$, saturated aqueous $\mathrm{NH}_{4} \mathrm{Cl}(2 \times 10 \mathrm{~mL})$, and saturated aqueous $\mathrm{NaCl}(2 \times 10 \mathrm{~mL})$. The organic layer was dried $\left(\mathrm{Na}_{2} \mathrm{SO}_{4}\right)$, filtered, concentrated, and the residue was purified by flash chromatography (silica gel, 5-10\% EtOAc/hexane) to afford rac-42 (0.262 g, 85\%) as a colorless solid: ${ }^{1} \mathrm{H} \mathrm{NMR}\left(\mathrm{CDCl}_{3}, 400 \mathrm{MHz}\right) \delta 6.93(\mathrm{~s}, 1 \mathrm{H}), 6.41(\mathrm{~s}, 1 \mathrm{H}), 5.93(\mathrm{~s}, 2 \mathrm{H}), 5.23(\mathrm{~d}, 1 \mathrm{H}, J=2.8$ Hz), 4.02 (s, 3H), 3.90 (s, 6H), 3.84 (s, 3H), 3.04 (dd, 1H, $J=13.6,3.2 \mathrm{~Hz}), 2.40$ (dd, 1H, $J=$ 13.2, $11.6 \mathrm{~Hz}), 1.93(\mathrm{~m}, 1 \mathrm{H}), 1.69(\mathrm{~m}, 1 \mathrm{H}), 0.98$ (d, 3H, $J=6.8 \mathrm{~Hz}), 0.94$ (d, 3H, $J=8.4 \mathrm{~Hz})$, $0.93(\mathrm{~s}, 9 \mathrm{H}), 0.09$ (s, 3H), -0.23 (s, 3H); ${ }^{13} \mathrm{C} \mathrm{NMR}\left(\mathrm{CDCl}_{3}, 100 \mathrm{MHz}\right) \delta 152.2,150.1,148.5$, $148.2,141.9,139.5,136.6,135.5,109.5,108.2,108.0,105.1,101.4,74.3,61.1,60.4,60.0,56.0$, 45.0, 40.0, 35.8, 25.9, 25.8, 21.0, 18.1, 17.1, 10.3, -4.6, -4.9; IR (film) $v_{\max } 2931,1604,1567$, 1477, 1394, 1252, 1105, 837, $776 \mathrm{~cm}^{-1}$; HRMS (ESI), $m / z$ calcd for $\mathrm{C}_{29} \mathrm{H}_{42} \mathrm{Br}_{2} \mathrm{O}_{7} \mathrm{SiNa}$ : 711.0964 ; found: 711.0992 .

1-(3,4,5-trimethoxyphenyl)-2,3-dimethylbut-3-en-1-one: A solution of (E)-2-methyl-2-butene1-ylmagnesium bromide in THF $(0.10 \mathrm{M}, 54 \mathrm{~mL}, 5.4 \mathrm{mmol})$ was added dropwise by syringe over $30 \mathrm{~min}$ to a solution of 3,4,5-trimethoxybenzaldehyde $(0.75,4.52 \mathrm{mmol})$ in THF $(10 \mathrm{~mL})$ at $-78^{\circ} \mathrm{C}$. The reaction mixture was stirred for $2 \mathrm{~h}$ at $-78^{\circ} \mathrm{C}$ and was quenched by the addition of saturated aqueous $\mathrm{NH}_{4} \mathrm{Cl}(10 \mathrm{~mL})$. The volatiles were removed and the residue was dissolved in EtOAc $(100 \mathrm{~mL})$ and was washed with water and saturated aqueous $\mathrm{NaCl}$. The organic layer was concentrated to afford 1-(3,4,5-trimethoxyphenyl)-2,3-dimethylbut-3-en-1-ol (crude wt. $0.96 \mathrm{~g}$, 
$3.6 \mathrm{mmol}$ ), which was used without purification. The alcohol was dissolved in ethyl acetate (15 $\mathrm{mL})$ under nitrogen atmosphere and IBX (2.18 g, $7.77 \mathrm{mmol})$ and DMSO (5 drops) were added. The reaction mixture was warmed at $55^{\circ} \mathrm{C}$ for $3 \mathrm{~h}$ and was cooled and washed with saturated sodium thiosulfate, saturated aqueous $\mathrm{NaCl}$, water. The organic layer was dried $\left(\mathrm{MgSO}_{4}\right)$, filtered, and concentrated and the residue was purified by flash chromatography $(10 \%$ EtOAc/hexane) to afford 1-(3,4,5-trimethoxyphenyl)-2,3-dimethylbut-3-en-1-one as a colorless solid (1.12 g, 89\%): ${ }^{1} \mathrm{H}$ NMR $\left(\mathrm{CDCl}_{3}, 400 \mathrm{MHz}\right) \delta 4.89$ (d, 2H, $\left.J=7.4 \mathrm{~Hz}\right), 4.09$ (q, $1 \mathrm{H}, J=6.7$ $\mathrm{Hz}$ ), 3.86 (s, 9H), 1.70 (s, 3H), 1.29 (d, 3H, $J=6.7) ;{ }^{13} \mathrm{C} \mathrm{NMR}\left(\mathrm{CDCl}_{3}, 100 \mathrm{MHz}\right) \delta 199.9$, 153.3, 146.2, 142.8, 132.3, 113.7, 106.4, 61.3, 56.6, 49.7, 20.7, 16.6; IR (film) $v_{\max } 2938,1678$, 1585, 1415, 1342, $1129 \mathrm{~cm}^{-1}$; HRMS (ESI), $m / z$ calcd for $\mathrm{C}_{15} \mathrm{H}_{20} \mathrm{O}_{4} \mathrm{Na}$ : 287.1259; found: 287.1260 .

1-(1 $\left.R^{*}, 2 R^{*}\right)-1-(3,4,5-$ trimethoxyphenyl)-2,3-dimethylbut-3-en-1-ol: A solution of the above ketone $(1.08 \mathrm{~g}, 4.09 \mathrm{mmol})$ in anhydrous methanol $(20 \mathrm{~mL})$ under nitrogen was cooled to $-78^{\circ} \mathrm{C}$ and treated with sodium borohydride $(0.23 \mathrm{~g}, 6.13 \mathrm{mmol})$ portionwise. The reaction mixture was stirred for $10 \mathrm{~h}$ at $-78{ }^{\circ} \mathrm{C}$ and quenched at that temperature by the addition of $\mathrm{AcOH}(0.36 \mathrm{~mL}$, $6.13 \mathrm{mmol})$. The solvent was removed and the residue was dissolved in EtOAc $(50 \mathrm{~mL})$ and washed with water and saturated aqueous $\mathrm{NaCl}$. The organic layer was dried $\left(\mathrm{MgSO}_{4}\right)$, filtered, and concentrated, and the residue was purified by flash chromatography (5-25\% EtOAc/hexane) to afford the anti-alcohol as a colorless solid $(0.92 \mathrm{~g}, 85 \%)$ : ${ }^{1} \mathrm{H}$ NMR $\left(\mathrm{CDCl}_{3}, 500 \mathrm{MHz}\right) \delta 6.58$ $(\mathrm{d}, 2 \mathrm{H}, J=2.4 \mathrm{~Hz}), 4.99(\mathrm{~d}, 2 \mathrm{H}, J=1.0 \mathrm{~Hz}), 4.30(\mathrm{~d}, 1 \mathrm{H}, J=9.5 \mathrm{~Hz}), 3.88(\mathrm{~s}, 3 \mathrm{H}), 3.87(\mathrm{~s}, 3 \mathrm{H})$, 3.84 (s, 3H), 2.50-2.40 (m, 1H), 2.28 (br s, $1 \mathrm{H}), 1.79$ (s, 3H), 0.82 (d, $3 \mathrm{H}, J=6.9 \mathrm{~Hz}$ ); ${ }^{13} \mathrm{C}$ NMR $\left(\mathrm{CDCl}_{3}, 125 \mathrm{MHz}\right) \delta 153.6,147.7,138.5,138.0,114.2,104.4,106.4,76.9,61.2,56.6,50.4,18.9$, 16.5; IR (film) $v_{\max } 3592,3542,2968,2839,1593,1508,1462,1423,1386,1326,1271,1238$,

1184, 1129, 1005, 903, 838, $745 \mathrm{~cm}^{-1}$; HRMS (ESI), $\mathrm{m} / z$ calcd for $\mathrm{C}_{15} \mathrm{H}_{22} \mathrm{O}_{4} \mathrm{Na}$ : 289.1416; found: 289.1418 .

\section{$\left(\left(1 R^{*}, 2 R^{*}\right)-1-(3,4,5-\right.$ trimethoxyphenyl)-2,3-dimethylbut-3-enyloxy)(tert}

butyl)dimethylsilane (rac-43). Imidazole $(0.11 \mathrm{~g}, 1.8 \mathrm{mmol})$ was added to a solution of 1 (3,4,5-trimethoxyphenyl)-2,3-dimethylbut-3-en-1-ol (0.15 g, $0.6 \mathrm{mmol})$ in DMF (5 mL) at $25^{\circ} \mathrm{C}$. 
After $15 \mathrm{~min}, t-\mathrm{BuMe} \mathrm{SiCl}_{2}(0.13 \mathrm{~g}, 0.48 \mathrm{mmol})$ was added and the reaction mixture was stirred for $14 \mathrm{~h}$ at this temperature. The reaction mixture was diluted with water $(10 \mathrm{~mL})$ and extracted with diethyl ether. The organic layer was washed with saturated aqueous $\mathrm{NaCl}$ and water, and was dried $\left(\mathrm{MgSO}_{4}\right)$, concentrated, and the residue was purified by flash chromatography $(5 \%$ EtOAc/hexane) to afford rac-43 $(0.17 \mathrm{~g}, 79 \%)$ as a syrup: ${ }^{1} \mathrm{H}$ NMR $\left(\mathrm{CDCl}_{3}, 500 \mathrm{MHz}\right) \delta 6.50$ (s, 2H), 4.77 (s, 1H), 4.69 (s, 1H), 4.42 (d, 1H, $J=7.3 \mathrm{~Hz}$ ), 3.85 (s, 9H), 2.40 (quintet, 1H, $J=$ $7.1 \mathrm{~Hz}), 1.73$ (s, 3H), 0.86 (s, 9H), $0.82(\mathrm{~d}, 3 \mathrm{H}, J=7.0 \mathrm{~Hz}), 0.02(\mathrm{~s}, 3 \mathrm{H}),-0.22(\mathrm{~s}, 3 \mathrm{H}) ;{ }^{13} \mathrm{C}$ NMR $\left(\mathrm{CDCl}_{3}, 125 \mathrm{MHz}\right) \delta 152.6,147.4,139.7,137.0,111.7,104.0,78.4$ 61.0, 56.1, 49.6, 25.7, 20.8, 18.2, 16.1, -4.6, -5.2; IR (film) $v_{\max } 3071,2958,2856,1645,1592,1506,1462,1421,1348$, 1326, 1270, 1234, 1127, 1090, 1006, 943, 891, 857, 837, 776, $694 \mathrm{~cm}^{-1}$; HRMS (ESI), $\mathrm{m} / \mathrm{z}$ calcd for $\mathrm{C}_{21} \mathrm{H}_{36} \mathrm{O}_{4} \mathrm{SiNa}$ : 403.2280; found: 403.2274 .

Primary alcohol rac-44. A solution of $\mathbf{4 3}(0.3 \mathrm{~g}, 0.79 \mathrm{mmol})$ in THF $(2 \mathrm{~mL})$ was cooled to $0{ }^{\circ} \mathrm{C}$, and a solution of 9-BBN in THF $(0.5 \mathrm{M}, 0 . \mathrm{mL}, 0.94 \mathrm{mmol})$ was added dropwise by syringe over $20 \mathrm{~min}$. The reaction mixture was allowed to warm to $25{ }^{\circ} \mathrm{C}$ over $3 \mathrm{~h}$, and was stirred at this temperature for $16 \mathrm{~h}$. The reactopm mixture was cooled to $0{ }^{\circ} \mathrm{C}$, and treated with $\mathrm{NaOH}(94 \mathrm{mg}$, $2.36 \mathrm{mmol})$ dissolved in mixture of $1 \mathrm{~mL}$ EtOH and $1 \mathrm{~mL}$ THF and aqueous $30 \% \mathrm{H}_{2} \mathrm{O}_{2}(0.13$ $\mathrm{mL}, 1.13 \mathrm{mmol}$ ). The reaction mixture was allowed to warm to $25{ }^{\circ} \mathrm{C}$. After $10 \mathrm{~h}$ at this temperature, the volatiles were removed and the residue was diluted with EtOAc $(25 \mathrm{~mL})$ and washed with water and saturated aqueous $\mathrm{NaCl}$. The organic layer was dried $\left(\mathrm{Na}_{2} \mathrm{SO}_{4}\right)$, concentrated, and the residue was purified by flash chromatography ( $30 \% \mathrm{EtOAc} / \mathrm{hexane})$ to afford rac-44 (0.28 g, 91\%) as a solid: ${ }^{1} \mathrm{H}$ NMR $\left(\mathrm{CDCl}_{3}, 400 \mathrm{MHz}\right) \delta 6.52(\mathrm{~s}, 2 \mathrm{H}), 4.64(\mathrm{~d}, 1 \mathrm{H}, J$ $=4.8 \mathrm{~Hz}), 3.84(\mathrm{~s}, 9 \mathrm{H}), 3.53(\mathrm{dd}, 1 \mathrm{H}, J=8.8,11.1 \mathrm{~Hz}), 3.31(\mathrm{dd}, 1 \mathrm{H}, J=4.9,5.5 \mathrm{~Hz}), 2.05-1.90$ (m, 1H), 1.85-1.80 (m, 1H), 1.25 (bs, 1H), $0.93(\mathrm{~s}, 12 \mathrm{H}), 0.84$ (d, 3H, J = 7.1 Hz), 0.09 (s, 3H), 0.13 (s, 3H); ${ }^{13} \mathrm{C} \mathrm{NMR}\left(\mathrm{CDCl}_{3}, 125 \mathrm{MHz}\right) \delta 152.8,139.1,136.7,103.7,79.1,64.0,61.0,56.1$, 45.7, 34.0, 25.8, 18.2, 17.3, 12.2, -4.6, -5.0; IR (film) $v_{\max } 3403,2959,2858,1593,1506,1463$, $1420,1329,1263,1235,1184,1127,1078,1054,1005,934,918,838,778,725,674 \mathrm{~cm}^{-1}$; HRMS (ESI), $m / z$ calcd for $\mathrm{C}_{21} \mathrm{H}_{38} \mathrm{O}_{5} \mathrm{SiNa}$ : 421.2380; found: 421.2355 .

(X-Ray Crystal structure provided [Coleman 1106]). 
$\left(\left(1 R^{*}, 2 S^{*}\right)-1-(3,4,5\right.$-trimethoxyphenyl)-2,3-dimethylbut-3-enyloxy)(tert-butyl)dimethylsilane

(rac-45). Following the procedure for the preparation of $\mathbf{4 3}$, reaction of imidazole $(60 \mathrm{mg}, 0.96$ $\mathrm{mmol})$ and $t$ - $\mathrm{BuMe} \mathrm{SiCl}_{2}(70 \mathrm{mg}, 0.90 \mathrm{mmol})$ with $\left(1 R^{*}, 2 S^{*}\right)-1-(3,4,5$-trimethoxyphenyl)-2,3dimethylbut-3-en-1-ol (80 mg, $0.32 \mathrm{mmol})$ in DMF $(3 \mathrm{~mL})$ at $25^{\circ} \mathrm{C}$ for $14 \mathrm{~h}$ afforded $\mathrm{rac}-45(90$ mg, $76 \%$ ) as a syrup: ${ }^{1} \mathrm{H} \mathrm{NMR}\left(\mathrm{CDCl}_{3}, 400 \mathrm{MHz}\right) \delta 6.50$ (s, 2H), 4.73 (s, 1H), 4.65 (s, 1H), $4.49(\mathrm{~d}, 1 \mathrm{H}, J=5.9 \mathrm{~Hz}), 3.84(\mathrm{~s}, 9 \mathrm{H}), 2.30$ (quintet, $1 \mathrm{H}, J=6.7 \mathrm{~Hz}), 1.60$ (s, 3H), 1.05 (d, 3H, $J$ $=6.9 \mathrm{~Hz}), 0.90(\mathrm{~s}, 9 \mathrm{H}), 0.02(\mathrm{~s}, 3 \mathrm{H}),-0.18(\mathrm{~s}, 3 \mathrm{H}) ;{ }^{13} \mathrm{C} \mathrm{NMR}\left(\mathrm{CDCl}_{3}, 100 \mathrm{MHz}\right) \delta 152.8,147.6$, $140.7,137.0,112.0,104.0,78.2,61.2,56.4,50.0,26.0,21.1,18.5,16.4,-4.5,-4.9$; IR (film) $v_{\max } 3048,2959,2856,1592,1461,1427,1326,1279,1235,1127,1090,1006,943,856,837$ $\mathrm{cm}^{-1}$; HRMS (ESI), $m / z$ calcd for $\mathrm{C}_{21} \mathrm{H}_{36} \mathrm{O}_{4} \mathrm{SiNa}$ : 403.2281; found: 403.2307.

\section{$\left(1 R^{*}, 2 S *, 3 S^{*}\right)-4-(3-(B e n z y l o x y)-4,5-d i m e t h o x y p h e n y l)-1-(3,4,5-t r i m e t h o x y p h e n y l)-2,3-$}

dimethylbutoxy)(tert-butyl)dimethylsilane (rac-47). 9-Borabicyclo[3.3.1]nonane (0.15 g, 1.26 mmol) was added dropwise at $0{ }^{\circ} \mathrm{C}$ over $30 \mathrm{~min}$ to a solution of alkene rac-45 $(0.40 \mathrm{~g}, 1.05$ $\mathrm{mmol})$ in THF (3.0 mL). The reaction mixture was allowed to warm to $25^{\circ} \mathrm{C}$ and was stirred for $12 \mathrm{~h}$ before being cooled to $0{ }^{\circ} \mathrm{C}$. The alkylborane formed was added dropwise using a syringe over $15 \mathrm{~min}$ to a mixture of aryl bromide $46(0.26 \mathrm{~g}, 1.05 \mathrm{mmol}), \mathrm{Ph}_{3} \mathrm{P}(0.04 \mathrm{~g}, 0.16 \mathrm{mmol})$, $\mathrm{Pd}\left(\mathrm{PPh}_{3}\right)_{4}(0.07 \mathrm{~g}, 0.06 \mathrm{mmol})$, and aqueous $\mathrm{NaOH}(4 \mathrm{M} 0.87 \mathrm{~mL}, 3.47 \mathrm{mmol})$ in THF $(5 \mathrm{~mL})$ at $0{ }^{\circ} \mathrm{C}$. The pale yellow reaction mixture was warmed at $70^{\circ} \mathrm{C}$ for $8 \mathrm{~h}$. The volatiles were removed and the residue was dissolved in $\mathrm{CH}_{2} \mathrm{Cl}_{2}(8 \mathrm{~mL})$ and was passed through a pad of Celite. Water $(2 \mathrm{~mL})$ was added to the filtrate followed by dropwise addition of $5 \%$ aqueous $\mathrm{HCl}$ until neutral. The mixture was washed with water $(2 \times 15 \mathrm{~mL})$, dried $\left(\mathrm{MgSO}_{4}\right)$, filtered, and concentrated, and the residue was purified by flash chromatography (silica, 5-25\% EtOAc/hexane) to afford the Suzuki product rac-47 as a colorless solid (0.45 g, 70\%), which was recrystallized from hot hexane: ${ }^{1} \mathrm{H}$ NMR $\left(400 \mathrm{MHz}, \mathrm{CDCl}_{3}\right) \delta$ 7.44-7.31 (m, 5H), $6.55(\mathrm{~s}, 2 \mathrm{H}), 6.29(\mathrm{~d}, 1 \mathrm{H}, J=1.6 \mathrm{~Hz})$, $6.21(\mathrm{~d}, 1 \mathrm{H}, J=1.6 \mathrm{~Hz}), 5.04(\mathrm{~d}, 2 \mathrm{H}, J=6.1 \mathrm{~Hz}), 4.53$ (d, 1H, $J=6.9 \mathrm{~Hz}), 3.85$ (s, 9H), 3.77 (s, $6 \mathrm{H}), 2.75(\mathrm{dd}, 1 \mathrm{H}, J=13.4,3.2 \mathrm{~Hz}), 2.04(\mathrm{~m}, 1 \mathrm{H}), 1.86(\mathrm{~m}, 1 \mathrm{H}), 1.67(\mathrm{~m}, 1 \mathrm{H}), 1.02(\mathrm{~d}, 3 \mathrm{H}, J=$ $6.7 \mathrm{~Hz}), 0.89(\mathrm{~s}, 9 \mathrm{H}), 0.81(\mathrm{~d}, 3 \mathrm{H}, J=6.6 \mathrm{~Hz}), 0.05(\mathrm{~s}, 3 \mathrm{H}),-0.20(\mathrm{~s}, 3 \mathrm{H}) ;{ }^{13} \mathrm{C} \mathrm{NMR}(125 \mathrm{MHz}$, $\left.\mathrm{CDCl}_{3}\right) \delta 153.5,153.3,152.4,140.9,137.7,137.5,137.2,128.8,128.1,127.7,108.7,106.5$, $104.4,78.6,71.5,61.3$ (2C), 56.6, 56.4, 47.6, 38.5, 35.9, 26.3, 18.8, 18.7, 11.5, -3.9, -4.5; IR 
(neat) $v_{\max } 2932,2855,1590,1506,1461,1327,1235,1124,836 \mathrm{~cm}^{-1}$; HRMS (ESI), $\mathrm{m} / z$ calcd for $\mathrm{C}_{36} \mathrm{H}_{52} \mathrm{O}_{7} \mathrm{SiNa}$ : 647.3375; found: 647.3371 .

(X-Ray Crystal structure provided [Coleman 1211]).

Dibenzocyclooctadiene 50. A solution of $n-\mathrm{Bu}_{4} \mathrm{NF}(56 \mathrm{mg}, 0.21 \mathrm{mmol})$ in THF $(2 \mathrm{~mL})$ was added to a solution of $49(25 \mathrm{mg}, 0.048 \mathrm{mmol})$ in THF $(2 \mathrm{~mL})$ at $55^{\circ} \mathrm{C}$ and the reaction mixture was stirred at this temperature for $12 \mathrm{~h}$. The volatiles were removed and the residue was dissolved in EtOAc $(10 \mathrm{~mL})$, and was washed with water and saturated aqueous $\mathrm{NaCl}$. The organic layer was dried $\left(\mathrm{Na}_{2} \mathrm{SO}_{4}\right)$, concentrated, and the residue was purified by flash chromatography (30\% EtOAc/hexane) to afford $\mathbf{5 0}(19 \mathrm{mg}, 89 \%)$ as colorless solid: ${ }^{1} \mathrm{H}$ NMR $\left(\mathrm{CDCl}_{3}, 500 \mathrm{MHz}\right) \delta 6.91(\mathrm{~s}, 1 \mathrm{H}), 6.45(\mathrm{~s}, 1 \mathrm{H}), 5.98(\mathrm{ABq}, 2 \mathrm{H}, J=1.5 \mathrm{~Hz}, \Delta v=6.6 \mathrm{~Hz}), 5.94$ $(\mathrm{ABq}, 2 \mathrm{H}, J=1.4 \mathrm{~Hz}, \Delta v=3.0 \mathrm{~Hz}), 4.52(\mathrm{~d}, 1 \mathrm{H}, J=1.4 \mathrm{~Hz}), 3.87$ (s, 3H), 3.85 (s, 3H), 2.12 (dd, $1 \mathrm{H}, J=13.4,9.5 \mathrm{~Hz}), 2.05-1.80(\mathrm{~m}, 3 \mathrm{H}), 1.54(\mathrm{br} \mathrm{s}, 1 \mathrm{H}), 1.00$ (d, 3H, $J=7.2 \mathrm{~Hz}), 0.70$ (d, $3 \mathrm{H}, J=7.1 \mathrm{~Hz}) ;{ }^{13} \mathrm{C} \mathrm{NMR}\left(\mathrm{CDCl}_{3}, 125 \mathrm{MHz}\right) \delta 149.1,148.3,140.7,140.6,138.2,135.5,135.2$, 134.3, 120.4, 119.3, 103.0, 102.1, 100.9, 100.8, 73.3, 59.7, 59.6, 42.5, 39.2, 34.6, 21.9, 14.1, 7.8; IR (film) $v_{\max } 3470,2956,2882,1632,1608,1472,1405,1277,1252,1132,1046,960,934,832$, $736,674 \mathrm{~cm}^{-1}$; HRMS (ESI), $m / z$ calcd for $\mathrm{C}_{22} \mathrm{H}_{24} \mathrm{O}_{7} \mathrm{Na}$ : 423.1420 ; found: 423.1427 .

Dibenzocyclooctadiene 51. A solution of dibromo-1,4-diarylbutane $\mathbf{X}(0.20 \mathrm{~g}, 0.28 \mathrm{mmol})$ in MeTHF $(5 \mathrm{~mL})$ was treated with $t$-BuLi $(2.3 \mathrm{M}, 0.49 \mathrm{~mL}, 1.13 \mathrm{mmol})$ at $-78{ }^{\circ} \mathrm{C}$ under argon. After $15 \mathrm{~min}$, the pale yellow reaction mixture was transferred via cannula to a flask containing $\mathrm{CuCN}(25 \mathrm{mg}, 0.28 \mathrm{mmol})$ in MeTHF $(2 \mathrm{~mL})$ at $-78{ }^{\circ} \mathrm{C}$ under argon. The heterogeneous mixture was allowed to warm to $-40^{\circ} \mathrm{C}$ over $1 \mathrm{~h}$, and was stirred until homogeneous at this temperature. The reaction mixture was treated with a freshly prepared solution of 1,3-dinitrobenzene $(0.5 \mathrm{M}$ in MeTHF, $2.26 \mathrm{~mL}, 1.13 \mathrm{mmol}$ ) at $-40{ }^{\circ} \mathrm{C}$ and was allowed to warm to $25{ }^{\circ} \mathrm{C}$. The reaction mixture was stirred at this temperature for $12 \mathrm{~h}$ and quenched by the addition of $10 \% \mathrm{NH}_{4} \mathrm{OH}$ in saturated aqueous $\mathrm{NH}_{4} \mathrm{Cl}$. The resulting two-phase mixture was stirred for $30 \mathrm{~min}$, and then separated; the aqueous phase was extracted two times with EtOAc $(20 \mathrm{~mL})$. The combined organic extracts were washed with water and saturated aqueous $\mathrm{NaCl}$. The organic layer was dried $\left(\mathrm{Na}_{2} \mathrm{SO}_{4}\right)$, concentrated, and the residue was purified by flash chromatography $(5 \%$ EtOAc/hexane) to afford $\mathbf{5 1}(88 \mathrm{mg}, 57 \%)$ as colorless solid: ${ }^{1} \mathrm{H} \mathrm{NMR}\left(\mathrm{CDCl}_{3}, 500 \mathrm{MHz}\right) \delta 7.02$ 
(s, 1H), $6.50(\mathrm{~s}, 1 \mathrm{H}), 4.42(\mathrm{~s}, 1 \mathrm{H}), 3.90(\mathrm{~s}, 3 \mathrm{H}), 3.89(2 \mathrm{~s}, 6 \mathrm{H}), 3.62(\mathrm{~s}, 3 \mathrm{H}), 3.60(2 \mathrm{~s}, 3 \mathrm{H}), 2.16$ $(\mathrm{dd}, 1 \mathrm{H}, J=13.2,9.1 \mathrm{~Hz}), 1.89(\mathrm{~d}, 1 \mathrm{H}, J=13.2 \mathrm{~Hz}), 1.95-1.80(\mathrm{~m}, 2 \mathrm{H}), 1.02(\mathrm{~d}, 3 \mathrm{H}, J=6.7 \mathrm{~Hz})$, $0.86(\mathrm{~s}, 9 \mathrm{H}), 0.66(\mathrm{~d}, 3 \mathrm{H}, J=6.7 \mathrm{~Hz}),-0.11(\mathrm{~s}, 3 \mathrm{H}),-0.20(\mathrm{~s}, 3 \mathrm{H}) ;{ }^{13} \mathrm{C} \mathrm{NMR}\left(\mathrm{CDCl}_{3}, 125 \mathrm{MHz}\right)$ $\delta 153.1,151.7,151.3,150.6,140.1,139.7,139.2,136.8,120.8,120.6,107.1,106.8,73.8,100.6$, $61.0,60.8,60.5,55.8,55.7,44.8,39.4,34.9,25.8,22.3,18.0,7.7,-4.8,-5.1$; IR (film) $v_{\max }$ 2958, 2934, 2856, 1596, 1487, 1461, 1402, 1363, 1322, 1270, 1235, 1198, 1105, 1048, 1006, 885, 837, 679, $656 \mathrm{~cm}^{-1}$; HRMS (ESI), $m / z$ calcd for $\mathrm{C}_{30} \mathrm{H}_{46} \mathrm{O}_{7} \mathrm{SiNa}$ : 569.2905; found: 569.2901. (X-Ray Crystal structure provided [Coleman 1300]).

Dibenzocyclooctadienes 52 and 53. A solution of $42(0.10 \mathrm{~g}, 0.15 \mathrm{mmol})$ in MeTHF (3.5 mL) was treated with $t$ - $\mathrm{BuLi}(2.5 \mathrm{M}, 0.23 \mathrm{~mL}, 0.58 \mathrm{mmol})$ at $-78{ }^{\circ} \mathrm{C}$ under argon. After $30 \mathrm{~min}$ the pale yellow reaction mixture was transferred via cannula to a flask containing $\mathrm{CuCN}$ (13 mg, $0.15 \mathrm{mmol})$ in MeTHF $(10 \mathrm{~mL})$ at $-78^{\circ} \mathrm{C}$ under argon. The heterogeneous reaction mixture was allowed to warm to $-40{ }^{\circ} \mathrm{C}$ over $1.5 \mathrm{~h}$, and was stirred until homogeneous. The reaction mixture was cooled to $-125{ }^{\circ} \mathrm{C}$ (liquid $\mathrm{N}_{2} / n$-pentane), and oxygen gas was bubbled in through a fritted glass tube for $4 \mathrm{~h}$. The reaction mixture was purged with argon, and placed under high vacuum for 1 min to remove dissolved oxygen. The reaction was warmed to $-30{ }^{\circ} \mathrm{C}$, and was quenched by the addition of a solution of $10 \% \mathrm{NH}_{4} \mathrm{OH}$ in saturated aqueous $\mathrm{NH}_{4} \mathrm{Cl}(6 \mathrm{~mL})$. The two-phase mixture was stirred for $30 \mathrm{~min}$ as it was allowed to warm to room temperature. The organic layer was separated, and the aqueous phase was extracted with EtOAc $(2 \times 20 \mathrm{~mL})$. The combined organics were washed with water $(10 \mathrm{~mL})$ and saturated aqueous $\mathrm{NaCl}(2 \times 10 \mathrm{~mL})$, and were dried $\left(\mathrm{Na}_{2} \mathrm{SO}_{4}\right)$, filtered, concentrated, and the residue was purified by flash chromatography (silica gel, 5\% EtOAc/hexane) to afford 52 and 53 (combined yield: $30 \mathrm{mg}, 39 \%$ ) as a colorless oil. $(P)-52$ (10 mg, 13\%) was characterized: ${ }^{1} \mathrm{H}$ NMR $\left(\mathrm{CDCl}_{3}, 500 \mathrm{MHz}\right) \delta 6.46(\mathrm{~s}, 1 \mathrm{H}), 6.37(\mathrm{~s}$, 1H), $5.90(\mathrm{ABq}, 2 \mathrm{H}, J=1.5 \mathrm{~Hz}, \Delta v=19.4 \mathrm{~Hz}), 4.46$ (br s, 1H), 3.90 (s, 3H), 3.89 (s, 3H), 3.88 (s, 3H), 3.52 (s, 3H), 2.14 (m, 1H), 2.05-1.95 (br m, 2H), 1.72-1.67 (br m, 1H), 0.92 (d, 3H, J= $6.95 \mathrm{~Hz}), 0.77(\mathrm{~d}, 3 \mathrm{H}, J=6.3 \mathrm{~Hz}), 0.71(\mathrm{~s}, 9 \mathrm{H}),-0.04(\mathrm{~s}, 3 \mathrm{H}),-0.26(\mathrm{~s}, 3 \mathrm{H}) ;{ }^{13} \mathrm{C} \mathrm{NMR}\left(\mathrm{CDCl}_{3}\right.$, $125 \mathrm{MHz}) \delta 152.2,151.1,148.3,141.3$ (2C), 135.5, 133.9, 121.9, 101.9, 100.4, 81.9, 60.9, 60.4, 59.1, 56.0, 40.8, 25.8 (2C), 18.1, -4.8, -5.1; IR (film) $v_{\max } 2953,1618,1473,1107,1056,835$ $\mathrm{cm}^{-1}$; HRMS (ESI), $\mathrm{m} / z$ calcd for $\mathrm{C}_{29} \mathrm{H}_{42} \mathrm{O}_{7} \mathrm{SiNa}$ : 553.2598; found: 553.2604. (M)-53 (20 mg, 26\%) was characterized: ${ }^{1} \mathrm{H}$ NMR $\left(\mathrm{CDCl}_{3}, 400 \mathrm{MHz}\right) \delta 6.86(\mathrm{~s}, 1 \mathrm{H}), 6.39(\mathrm{~s}, 1 \mathrm{H}), 5.95(\mathrm{ABq}$, 
$2 \mathrm{H}, J=1.4 \mathrm{~Hz}, \Delta v=16.1 \mathrm{~Hz}), 4.17(\mathrm{~d}, 1 \mathrm{H}, J=8 \mathrm{~Hz}), 3.92(\mathrm{~s}, 3 \mathrm{H}), 3.90(\mathrm{~s}, 3 \mathrm{H}), 3.82(\mathrm{~s}, 3 \mathrm{H})$, $3.64(\mathrm{~s}, 3 \mathrm{H}), 2.57(\mathrm{dd}, 1 \mathrm{H}, J=15.2,4.4 \mathrm{~Hz}), 2.35(\mathrm{dd}, 1 \mathrm{H}, J=15.6,10.4 \mathrm{~Hz}), 1.61(\mathrm{~m}, 2 \mathrm{H}), 0.94$ $(\mathrm{d}, 3 \mathrm{H}, J=6.8 \mathrm{~Hz}), 0.87(\mathrm{~s}, 9 \mathrm{H}), 0.78(\mathrm{~d}, 3 \mathrm{H}, J=6.4 \mathrm{~Hz}),-0.14(\mathrm{~s}, 3 \mathrm{H}),-0.23(\mathrm{~s}, 3 \mathrm{H}) ;{ }^{13} \mathrm{C} \mathrm{NMR}$ $\left(\mathrm{CDCl}_{3}, 125 \mathrm{MHz}\right) \delta 153.0,151.1,148.1,140.9,140.8,139.9,134.5,120.8,120.1,104.7,103.0$, 100.7, 74.3, 61.0, 60.8, 59.3, 55.7, 47.9, 40.1, 33.3, 25.9 (2C), 18.9, 18.1, 12.8, -4.6, -5.2; IR (film) $v_{\max } 2955,2358,1597,1464,1108,1054,836 \mathrm{~cm}^{-1}$; HRMS (ESI), $\mathrm{m} / z$ calcd for $\mathrm{C}_{29} \mathrm{H}_{42} \mathrm{O}_{7} \mathrm{SiNa}$ : 553.2598; found: 553.2604.

6-Bromo-5-hydroxypiperonal (62). A solution of 5-hydroxypiperonal 61 (2.0 g, $12.0 \mathrm{mmol})$ in dioxane $(80 \mathrm{~mL})$ was cooled to $15^{\circ} \mathrm{C}$. A solution of $N$-bromosuccinimide $(2.14 \mathrm{~g}, 12.0 \mathrm{mmol})$ in dioxane $(20 \mathrm{~mL})$ was added dropwise by syringe over $2 \mathrm{~h}$ at this temperature. The reaction mixture was stirred for $12 \mathrm{~h}$ between $15^{\circ} \mathrm{C}$ to $19{ }^{\circ} \mathrm{C}$ and was quenched by the addition of saturated aqueous $\mathrm{Na}_{2} \mathrm{~S}_{2} \mathrm{O}_{3}(10 \mathrm{~mL})$. The mixture was concentrated, and the residue was dissolved in EtOAc $(100 \mathrm{~mL})$ and washed with saturated aqueous $\mathrm{Na}_{2} \mathrm{~S}_{2} \mathrm{O}_{3}$, water, and saturated aqueous $\mathrm{NaCl}$. The organic layer was dried $\left(\mathrm{Na}_{2} \mathrm{SO}_{4}\right)$, concentrated, and the residue was purified by flash chromatography ( silica, 15\% EtOAc/hexane) to afford pure 62 (2.84 g, 97\%) as a solid: ${ }^{1} \mathrm{H}$ NMR (DMSO-d $\left.6,500 \mathrm{MHz}\right) \delta 10.75$ (s, 1H), 10.12 (s, 1H), $6.94(\mathrm{~s}, 1 \mathrm{H}), 6.19(\mathrm{~s}, 2 \mathrm{H}) ;{ }^{13} \mathrm{C}$ NMR (DMSO-d 6 , 125 MHz) $\delta 191.0,148.6,141.4$, 138.6, 128.2, 112.6, 103.3, 100.8; IR (KBr) $v_{\max } 3042,2362,1660,1594,1497,1480,1460,1416,1355,1256,1124,1083,1040,994,922$, 836, 747, $668 \mathrm{~cm}^{-1}$; HRMS (ESI), $m / z$ calcd for $\mathrm{C}_{8} \mathrm{H}_{5} \mathrm{BrO}_{4} \mathrm{Na}$ : 266.9269; found: 266.9270 .

6-Bromo-7-methoxybenzo $[d][1,3]$ dioxole-5-carbaldehyde (22). A mixture of 6-bromo-5hydroxypiperonal (62) (1.8 g, $7.4 \mathrm{mmol}), \mathrm{K}_{2} \mathrm{CO}_{3}(1.52 \mathrm{~g}, 11.0 \mathrm{mmol})$ and dimethyl sulfate (0.84 $\mathrm{mL}, 8.8 \mathrm{mmol})$ in acetone $(20 \mathrm{~mL})$ was heated at $56{ }^{\circ} \mathrm{C}$ for $2 \mathrm{~h}$. After cooling to room temperature, the mixture was filtered and concentrated. The residue was dissolved in EtOAc (50 $\mathrm{mL}$ ), and was washed with water and saturated aqueous $\mathrm{NaCl}$. The organic layer was dried $\left(\mathrm{Na}_{2} \mathrm{SO}_{4}\right)$, concentrated, and the residue was recrystallized from $\mathrm{CH}_{2} \mathrm{Cl}_{2} /$ hexane to afford pure 22 (1.62 g, 85\%) as a light yellow solid: ${ }^{1} \mathrm{H}$ NMR $\left(\mathrm{CDCl}_{3}, 500 \mathrm{MHz}\right) \delta 10.26(\mathrm{~s}, 1 \mathrm{H}), 7.16(\mathrm{~s}, 1 \mathrm{H})$, $6.09(\mathrm{~s}, 2 \mathrm{H}), 4.06(\mathrm{~s}, 3 \mathrm{H}) ;{ }^{13} \mathrm{C} \mathrm{NMR}\left(\mathrm{CDCl}_{3}, 125 \mathrm{MHz}\right) \delta 190.7,149.2,143.0,140.3,128.6$, 115.8, 103.2, 102.7, 60.4; IR (KBr) $v_{\max } 3042,2362,1660,1594,1497,1480,1460,1416,1355$, 
1256, 1124, 1083, 1040, 994, 922, 836, 747, $668 \mathrm{~cm}^{-1}$; HRMS (ESI), $m / z$ calcd for $\mathrm{C}_{9} \mathrm{H}_{7} \mathrm{BrO}_{4} \mathrm{Na}$ : 280.9424; found: 280.9425 .

3-(Benzyloxy)-5-bromo-1,2-dihydroxybenzene (65). A slurry of 3-benzyloxy-5-bromo-2hydroxybenzaldehyde $(64)(8.8 \mathrm{~g}, 28.6 \mathrm{mmol})$ in water $(150 \mathrm{~mL})$ was treated with $3 \mathrm{~N} \mathrm{NaOH}$ $(24 \mathrm{~mL}, 71.6 \mathrm{mmol})$ to afford pale yellow colored paste. The mixture was diluted with $50 \mathrm{~mL}$ water and treated dropwise with $30 \% \mathrm{H}_{2} \mathrm{O}_{2}(21 \mathrm{~mL}, 186.3 \mathrm{mmol})$ at $25{ }^{\circ} \mathrm{C}$ and the reaction mixture was stirred for $3 \mathrm{~h}$ until the yellow colored material became a free flowing solid. The reaction mixture was diluted with ethyl acetate $(150 \mathrm{~mL})$ and acidified to $\mathrm{pH} 2$ with $6(\mathrm{~N}) \mathrm{HCl}$. The organic layer was separated and washed with water and saturated aqueous $\mathrm{NaCl}$. The organic layer was dried $\left(\mathrm{Na}_{2} \mathrm{SO}_{4}\right)$, concentrated, and the residue was purified by flash chromatography (silica, 20\% EtOAc/hexane) to afford pure $65(7.01 \mathrm{~g}, 83 \%)$ as a solid: ${ }^{1} \mathrm{H}$ NMR $\left(\mathrm{CDCl}_{3}, 500 \mathrm{MHz}\right) \delta 7.45-7.35(\mathrm{~m}, 5 \mathrm{H}), 6.80(\mathrm{~d}, 1 \mathrm{H}, J=2.1 \mathrm{~Hz}), 6.71(\mathrm{~d}, 1 \mathrm{H}, J=2.1 \mathrm{~Hz}), 5.39$ (s, 1H), 5.37 (s, 1H), $5.06(\mathrm{~s}, 2 \mathrm{H}) ;{ }^{13} \mathrm{C} \mathrm{NMR}\left(\mathrm{CDCl}_{3}, 125 \mathrm{MHz}\right) \delta$ 146.6, 144.8, 135.7, 132.0, 128.8 (2 C), 128.7, 128.0 (2 C), 112.6, 111.7, 108.2, 71.7; IR (film) $v_{\max } 3054,2362,1496,1458$, 1257, 1122, 1087, 1042, 998, 836, $748 \mathrm{~cm}^{-1}$; HRMS (ESI), $\mathrm{m} / z$ calcd for $\mathrm{C}_{13} \mathrm{H}_{11} \mathrm{BrO}_{3} \mathrm{Na}$ : 316.9789; found: 316.9780 . 


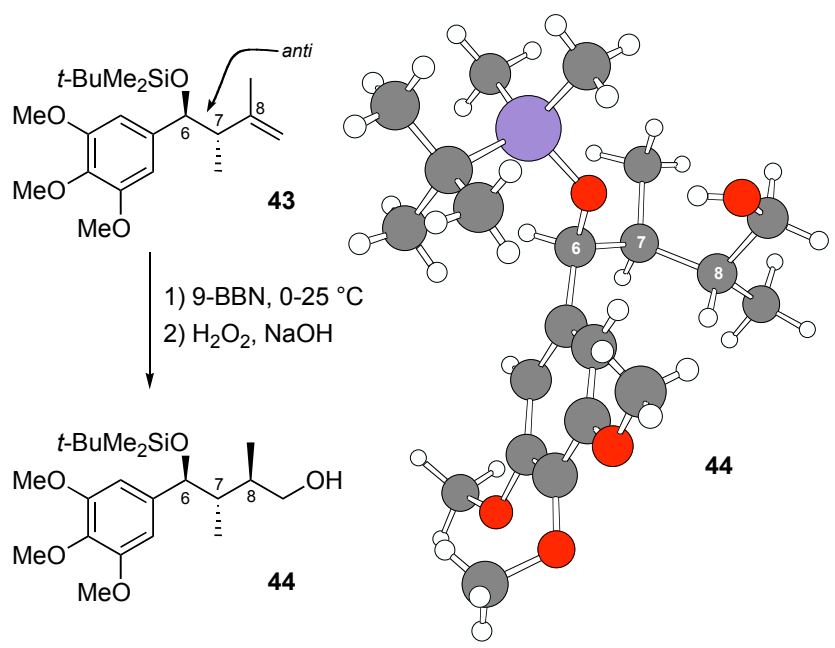

X-ray crystal structure of 44 .

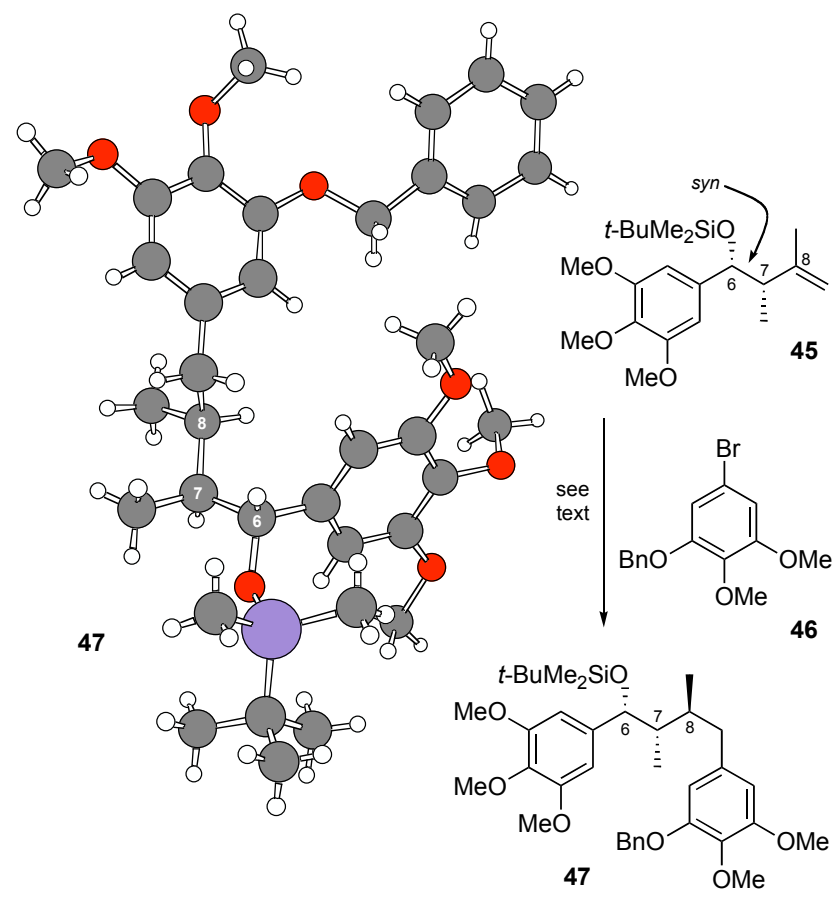

X-ray crystal structure of 47 .

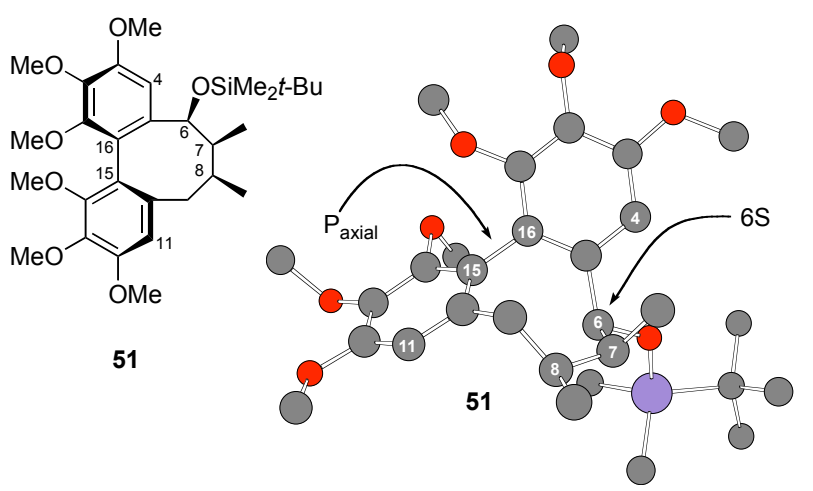

X-ray crystal stucture of $(P)-\mathbf{5 1}$. 


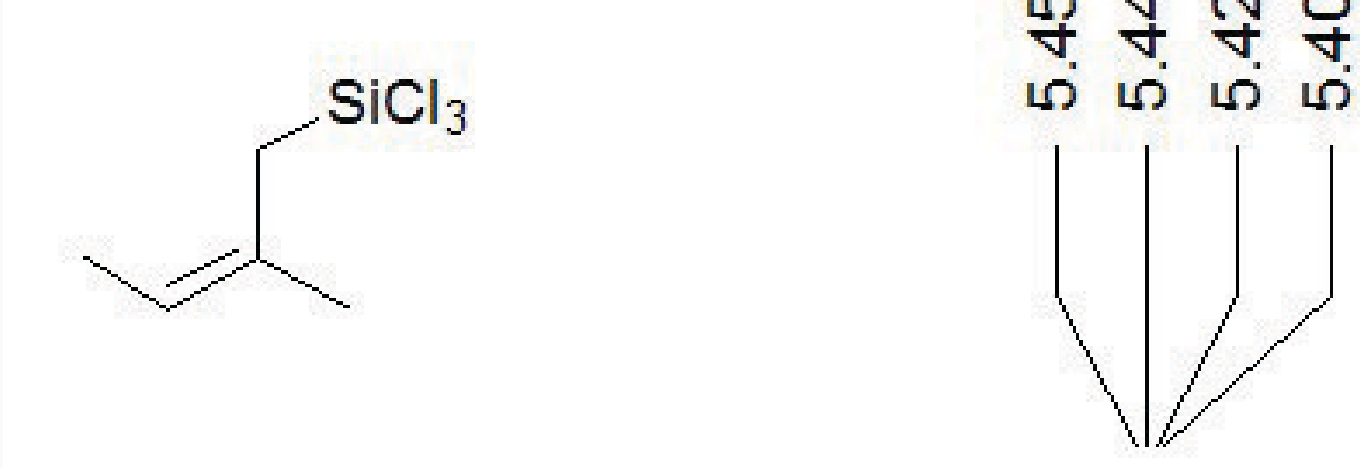

ֻ
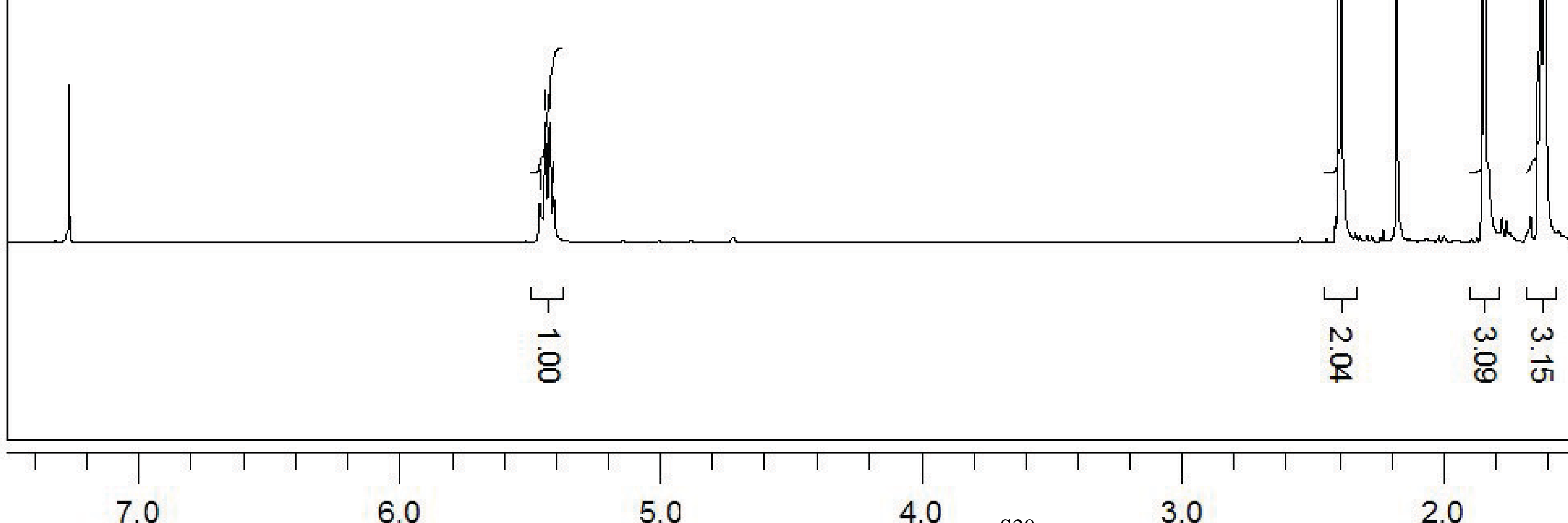
$\Delta \mid \mathrm{ppm}$ (f1)
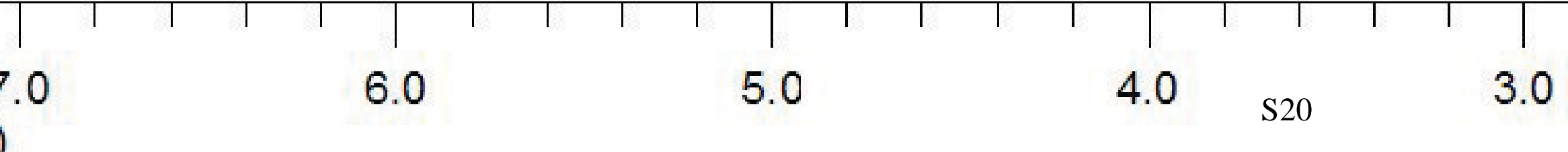
$-40000$

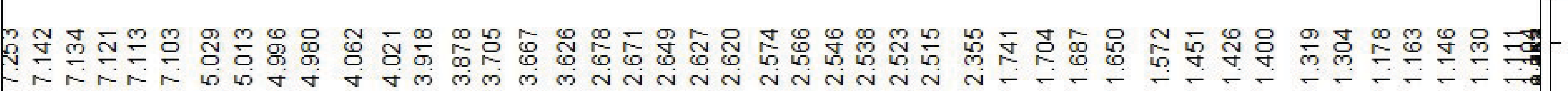

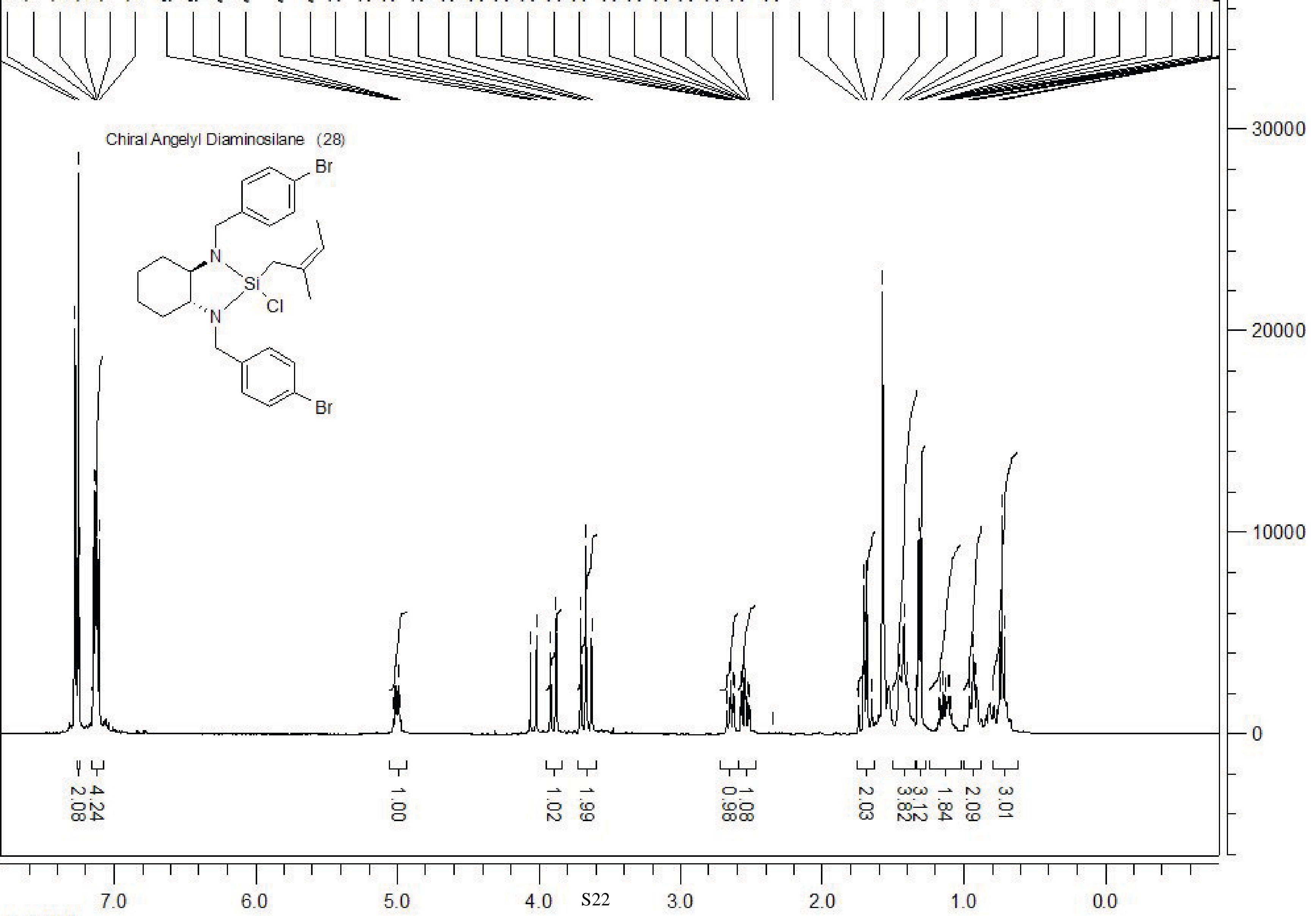


오윰ำ

$\min$

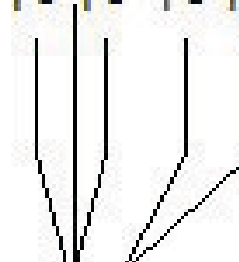

Chiral Tiglyldiamino Silane (29)

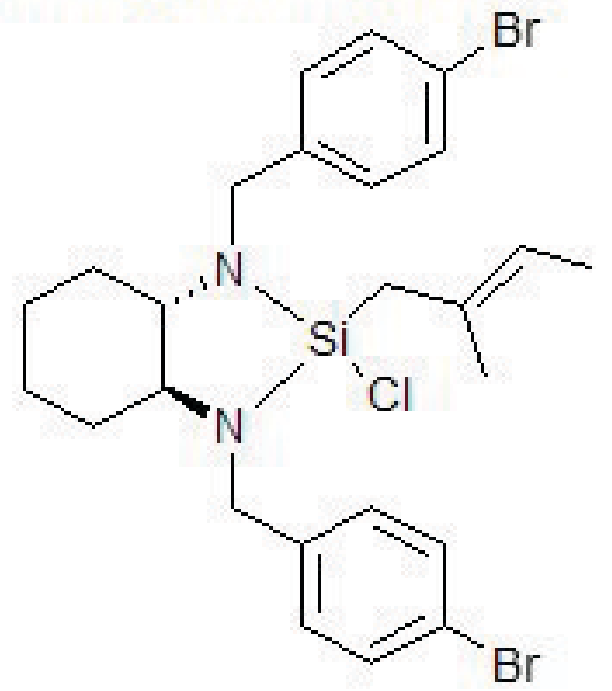

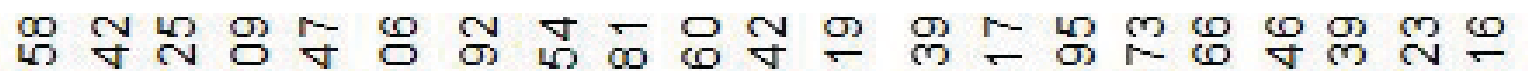

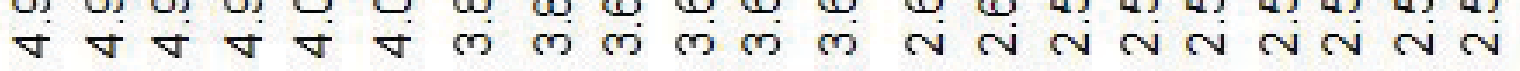
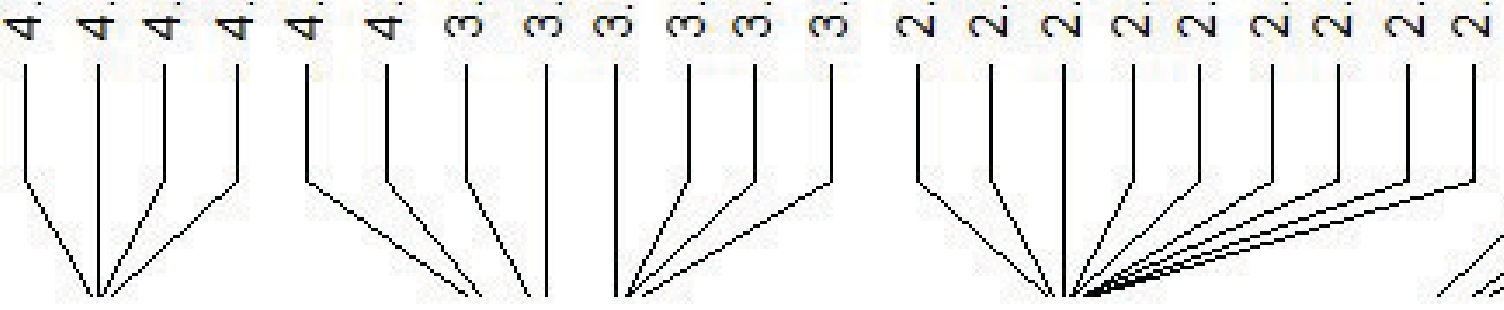
舫

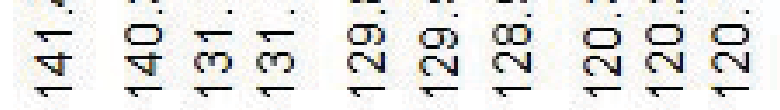

Tiglyl Chiral Amine C-13 (29)

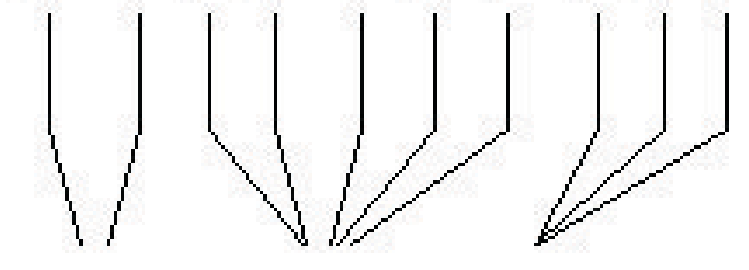

市

0

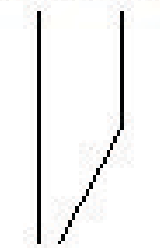

$\stackrel{\infty}{\infty}$

$\infty$

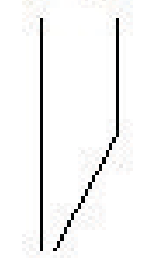

เᄄ山

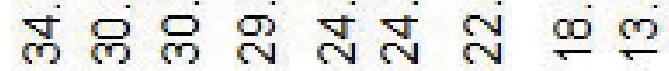

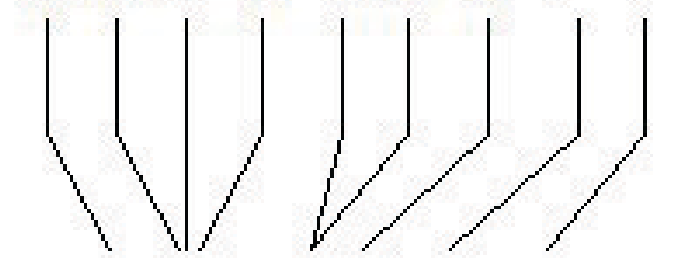

